NYPL RESEARCH LIBRARIES

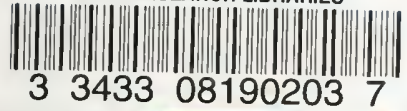



IQ

$(-1)+5064)$

$$
\text { EMIs }
$$







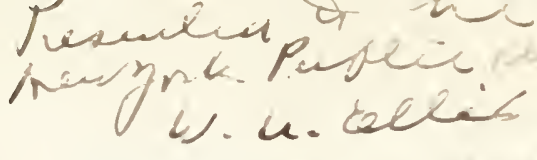

Index

$65.95: 6: 9$

\section{HISTORY OF VERMONT}

\section{NATURAL, CIVIL AND STATISTICAL}

by ZADOCK THOMPSON, A. M.

\section{EDITION OF 1842}

COMPILED BY

WILLIAM ARBA ELLIS, A. M. 



\title{
Index
}

\section{HISTORY OF VERMONT}

NATURAL, GIVIL AND STATISTICAL by ZADOCK THOMPSON, A. M. EDITION OF 1842

\author{
COMPILED BY \\ WILLIAM ARBA ELLIS, A. M.
}

Copyrightén BY

The Vermont HistcricAL" SOCItT

1913\%

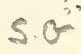



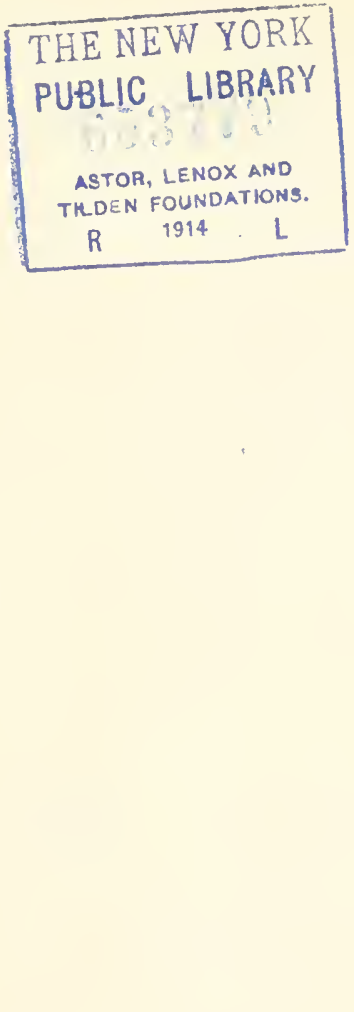


\title{
Index
}

\section{History of Vermont}

\author{
FOREWORD.
}

Mr. Marcus D. Gilman states in his valuable work, "The Bibliography of Vermont" in regard to the work of Mr. Zadock Thompson:

"We publish at the expense of the State of Vermont glowing eulogies upon our ephemeral politicians, such as members of Congress, etc., and upon our military heroes, as we call them, and yet the services of such a man as Mr. Thompson will endure in the grateful memory of succeeding generations long after the classes named have passed into oblivion and be remembered no more." Zadock Thompson's "History of Vermont" has been for years a standard work on the history of our State. This old leather bound book, now musty with age, has ever been an unfailing spring of information, bubbling with historic facts to delight the minds of the youth of the State. What a pleasure it was to many of us in our youth to read from its pages, yellowed with age, the "h?story of our State. His accounts of the Tndian defreditions made the facts he records a living prescit; ; and in iancy we saw the Indian skulking in our forest's inf heard his shrill war cry ring out in the calm of the pight. 'His record of the heroes of the early days fired our 'arts' with a loyalty for the State and inspired us with a spirit of emulation. 
As the compiler of this index has read the history written by Mr. Thompson, he has been impressed with the great wealth of historical facts stored in the pages of the work; and it is his desire that the index, herewith transmitted to the Society, will be of material assistance to the historian and the general reader and that it will make the history more useful for a ready work of reference. The compiler also wishes to acknowledge the valuable service rendered him by G. Harold Ellis, formerly of Northfield, for his assistance in the work.

Wilitiam A. Elilis.

East Orange, N. J., February 27, 1913.

\section{REVEREND ZADOCK THOMPSON, A. M.}

One of the ablest historians and naturalists of Vermont was the Rev. Zadock Thompson, born in Bridgewater, Vermont, May 23, I796, and son of Barnabas and Sarah (Fuller) Thompson.

The Thompson family is of Welsh descent and trace their ancestry to John Thompson, (or Thomson) as he spelled his name, born in the North of Wales in r6r6. About 1636 he emigrated to America, settling at Plymouth, Mass. He was a capable carpenter and in company with Richard Church built the first framed meeting house in Plymouth, in i637. He later removed to Sandwich where he purchased a fam. About I640 he purchased a large tract of land af Williani Weriş-pa-quin, a sachem of the Neponset tribe of indians about thirteen miles west of Plymouth tear the baindary lines of Bridgewater and Middleboro in what was then known as Plymouth, but now Halifax. Here he built his log cabin and cleared his farm. 
He at once took a prominent part in the civic and business life of the new town. During King Philip's war he was active in the military service of the colony. He was commissioned lieutenant-commander of the forces from his town and is said to have repeatedly saved the settlement at Halifax and Middleboro from the attacks of the Indians through "his superior skill and well timed caution."

Lieutenant Thompson became a prosperous farmer and was one of the most respected citizens of his town. He died in Halifax, June 16, 1696, and was buried in Middleboro.

He married December 26, I645, Mary Cooke, daughter of Francis Cooke, one of the "Pilgrim Fathers," who emigrated to Massachusetts in the Mayflower in I620. Mrs. Thompson was born in I626 and died March 21, I7I4. Eleven children were born to Lieut. John and Mary (Cooke) Thompson: I Adam, who died young. 2 John, born in I648, married Mary Tinkham. 3 Mary, born in I650, married Capt. Thomas Taber, and settled near Bedford, Mass. 4 Esther, born July 28, 1652, married William Reed. 5 Elizabeth, born Jan. 28, I654, married Thomas Swift, and settled in Nobscusset. 6 Sarah, born April 7, I657, died unmarried. 7 Lydia, born Oct. 5, I659, married James Soule. 8 Jacob, mentioned below. 9 Thomas, born Oct. I9, I664, married Mary Morton. Io Peter, married Rebecca Sturtevant. I I Mercy, born in I67I, died April I9, I756. Rev. Zadock Thompson's line of descent from John Thompson is as follows:

(II) Jacob, son of Lieut. John and Mary (Cooke) Thompson, was born in Halifax, Mass., April 24, I662 and died there Sept. I, I 726. He was, a prominent citizen of 
his native town. He married Abigail Wadsworth, who died Sept. I5, I774. Ten children.

(III) Barnabas, son of Jacob and Abigail (Wadsworth) Thompson, was born in Halifax, Jan. 28, I705, and died there December 20, I798. He married Hannah Porter, who died May 2, 1787.

(IV) Noah, son of Barnabas and Hannah (Porter) Thompson, was born in Halifax, March 20, I747. Soon after the close of the Revolutionary war he removed to Bridgewater, Vermont, where he made his home until his death May 5, I8I3. He was a highly respected citizen of the town and prominently identified with its history. $\mathrm{He}$ married Priscilla Holmes, who died April 9, I839. Ten children.

(V) Barnabas, son of Noah and Priscilla (Holmes) Thompson, was born in Halifax, Massachusetts, November 20, 1769 and died in Bridgewater, March 20, 1838. He removed to Vermont with his parents and made his home in Bridgewater until his death. He was a prominent citisen of the town. He took an active interest in military matters and served as captain in the state militia. He married Sarah Fuller. Five children: Barnabas, Zadock, mentioned below, Sally, Salmon and Eliza.

(VI) Rev. Zadock Thompson, after an academic education, entered the University of Vermont, and graduated in I823 with the degree of bachelor of arts. Later his alma mater, in recognition of his scholarly attainments, conferred upon him the degree of master of arts. During 1823-25, he served as tutor at the University. He then taught school in various towns in Vermont until I834, when he became principal of an academy in Hatley, 
Canada. During $1835-36$, he was principal of the Charleston Academy in Stanstead, Canada. He studied theology during his spare time and in May, 1837 he was ordained deacon in the Protestant Episcopal Church. He returned to Burlington in 1837 where he made his home until his death, January 19, I856. For a few years following I837 he was instructor at the Vermont Episcopal Institute in Burlington.

In early boyhood he had great love for all objects in nature. Nothing pleased him more than to wander in the fields and woods and study the plants and the habits of the animals. During his college course he made botany, zoology and geology his specialty, and in these subjects he became a recognized authority. During $1845-47$, he was assistant State Geologist and from 1853 until his death was the State Geologist. One of his great undertakings was the collecting of over 3,000 specimens of Vermont productions. He served as professor of natural history at the University of Vermont during I85I-56 and as professor of chemistry in 1851-54.

$\mathrm{He}$ was also an able mathematician and as early as I8rg began publishing an almanac. He made the astronomical observations for the Vermont Register for many years; and from 1822 until his death he was the astronomical editor of Walton's Vermont Register.

He was a prolific writer. His first book was a "Gazetteer of the State of Vermont, containing a brief General View of the State," a work containing an historical and topographical description of all the counties, towns and rivers; also a map and several cuts; 12 mo., 3ro pp., published by E. P. Walton in 1824 . 
His most important work and on which he worked for many years was his "History of Vermont, Natural, Civil and Statistical." This work was printed by Chauncey Goodrich in 1842 and contained three parts of 224, 224 and 200 pages, a map of the State and 200 cuts.

In 1853 he published his "Appendix to the History of Vermont, Natural and Statistical." The work was printed by Stacey and Jameson and contained 63 pages and one map. This appendix was also bound with the edition of 1842 and issued as a revised edition of the work.

His other works are: "History of Vermont from Its Settlement to the Close of the Year I832," I8 mo., 252 pp. ; also another edition of the same was printed by Smith and Harrington, Burlington, I836. "The Youth's Assistant in Practical Arithmetic designed for the use of Schools in the United States," 8 vo., I60 pp., David Watson, Woodstock, I825.

"The Youth's Assistant in Theoretic and Practical Arithmetic, designed for the use of Schools of the United States"; a second edition of this work with corrections and additions, David Watson, Woodstock; also another edition printed by E. \& T. Mills, Burlington, I828, a work of $58 \mathrm{pp}$.

"Thompson's New Arithmetic," 12 mo., 2 I6 pp., David Watson, Woodstock, 1828 .

"Thompson's New Arithmetic, the Youth's Assistant," I 2 mo., 216 pp., David Watson, Woodstock, 1829.

"Geography and History of Lower Canada, designed for the use of Schools," I2 mo., I 6 pp., map, Watson \& Gaylord, I 835 . 
"The Youth's Assistant in Theoretic and Practical Arithmetic," Ioth edition, I 2 mo., 168 pp., Vernon Harrington, Burlington, 1837 .

"Guide to Lake George, Lake Champlain, Montreal and Quebec," with map, tables of distances and route from Albany, Burlington, Montreal, etc., 24 mo., 48 pp., Burlington, 1845 .

"Geography and Geology of Vermont," with State and county outline maps for the use of schools and families, I2 mo., 2I 8 pp., Chauncey Goodrich, Burlington, I848.

"First Book of Geography for Vermont Children," 18 mo., 74 pp., C. Goodrich, Burlington, 1849.

"Natural History of Vermont," an address delivered at Boston before the Boston Society of Natural History in June, I85o. 8 vo., 32 pp., Chauncey Goodrich, Burlington, I 850 .

"Journal of a Trip to London, Paris and the Great Exhibition in I85I." I2 mo., I44 pp., published by Nichols \& Warren, George J. Stacey, printer.

"Northern Guide-Lake Champlain, Montreal and Quebec, Green and White Mountains and Willoughby Lake," with maps and tables and distances. I 8 mo., 56 pp., I854, published by S. B. Nichols, Burlington. Stacey \& Jameson, printers.

"Northern Guide, etc.," second edition of the preceding work I 2 mo., 45 pp., S. B. Nichols, Burlington, I 857 .

"History of the State of Vermont for the use of Families and Schools," I 2 mo., 252 pp. Smith \& Co. Burlington, 1858 .

He wrote many articles on history, geology and botany for various papers of the country. During 1828 he edited 
the Iris and Burlington Literary Gazette, and in 1832 the Green Mountain Repository.

Mr. Thompson was one of the ablest historians the State ever produced.

Mr. Gilman in his Bibliography of Vermont well says: "Mr. Thompson devoted the entire period of his life to the interests and welfare of his native State, and his labors are more gratefully appreciated as time passes. The best monument to his memory is his works. The people of Vermont have not publicly manifested that gratitude to the memory of Mr. Thompson which his labors merit."

He married, about I826, Phoebe Boyce. Three children were born of this marriage: Harriet F., born February, I827; Adaline Phoebe, born March, I829, and a child who died in infancy. 


\section{Index of Names}





\section{Index of Names}

A.

Abbey, Dillia (2) 222.

Abbott, Mr. (3) 79.

“ Rev. Benjamin (3) 24.

“ James (3) 124.

Abercrombie, General (2) 12-13, (3) 49.

Ackland, Major (2) 48.

Adams, Rev. C. (3) 125.

" Charles B. (1) 125-26, 151, 155.

“ Daniel (3) 22.

“ Elisha (3) 139.

“ Rev. Ephraim (2) 150.

“ James (2) 162, (3) 157.

“ John (2) 88-9, (3) 175.

“ John Quincy (2) 100.

“ Martin (3) 57.

“ Rev. Parker (3) 114, 176.

Alken, Edward (3) 2, 103, 193.

" John (3) 59.

Aikens, Asa (2) 124, 127.

" Solomon (3) 8.

Aldis, Asa (3) 123, 127, 155.

Aldrich, Mr. (3) 34, 137.

Alexander, E. (2) 167, 170.

“ John (3) 29.

“ Philip (3) 145.

Alger, Willis S. (2) 151.

Allen, Mr. (3) 3, 83

“ Barna (3) 93.

“ Benjamin (3) 184.

“ David (3) 154.

“ Ebenezer (3) 70, 126, 128, 143.

“ Elisha (3) 192.

“ Elizabeth (2) 173.

“ Ethan (2) $21-35,46,53-8,62-5,67,77-8,107,122,173$, (3) $4,16-17,31-2,49,82,93,129,163,169$.

“ Ezra (3) 190.

“ Frederick H. (2) 150.

“ Rev. George (2) 151, (3) 156.

“ Heber (3) 143 .

“ Heman (2) 49-51, 127, 149, 151, 223, (3) 140. 
Allen, Horace (2) 150.

“ Ira (2) $22,25,31-2,52,55,59,61,63-7,71,83,107,118-19$, $145,148,173-4,(3) 3,56,96,98$.

“ Ira H. (2) $58,149$.

“ J. A. (2) 167.

“ Rev. Jacob (3) 174.

“ Joel (2) 127.

“Jonathan (2) 162.

“ Justus (3) 34.

" Rev. Lewis (3) 145.

“ Samuel C. (2) 149.

“ Solomon M. (2) 156 .

Allyne, Abner (3) 12, 50.

"Jonathan (3) 12.

Amherst, General (2) 12-17, (3) 8, 49, 178.

Amy, Micah (3) 79.

Anderson, James (3) 109.

"William (2) $149,162$.

Andrews, Amos (3) 89.

“ Eldad (3) 59.

“ Rev. Elisha D. (3) 145.

“ Timothy (3) 91 .

Andrus, Benjamin (3) 190.

Angier, Rev. Aaron (3) 86.

Anthony, Mr. (2) 121.

“ Albro (3) 162.

“ John (3) 162.

Armand, John (3) 58.

Arms, John (3) 29.

“ Josiah (2) 73, 78.

“ Rev. Selah R. (3) 75, 194.

Armsby, Dr. James H. (2) 162.

Arnold, Benedict (2) $33-4,36-9$.

“Jonathan (2) 148, (3) 108, 157, 169.

“ Stephen (3) 55.

Arthur, William (3) 90.

Asheraft, Daniel (3) 81-2.

“ David (2) 78 .

“ Rev. N. B. (3) 129.

Ashley, Major (2) 10.

" Elisha (3) 116.

“ Enoch (3) 116.

“ Samuel (3) 194.

“ Thomas (3) 143.

Ashmin, Rev. Jehude (2) 150.

Aspenwall, Rev. Joseph C. (3) 61.

Atchinson, W. (2) 151.

Atherton, Joseph (3) 7.

Atklnson, Theodore (2) 224. 
Atlee, Mr. (2) 57.

Atwater, Rev. Jeremiah (2) 153, 155. "William (2) 150.

Atwood, Samuel (3) 14.

Austin, Apollos (2) 127.

“ Rev. John M. (2) 170.

“ Rev. Samuel (2) 102, 145-6, 148.

“ Seneca (2) 150.

" Seth (3) 174.

A verill, John (3) 128.

“ Samuel (3) 34.

Avery, Rev. David (3) 19.

"Rev. John A. (3) 116.

“ Rev. Royal A. (3) 44.

“ Samuel (3) 174.

Ayres, David (3) 36 .

" Miss E. (3) 64.

B.

Babbit, James (3) 98.

Babcock, Amos (3) 3.

“ John (3) 60.

“ Rev. Oliver W. (3) 67.

“ Solomon (3) 57.

Badger, Samuel (3) 52.

Bailey, General (2) 68-9.

"Benjamin (2) 150.

“ Benjamin F. (2) 150.

“ Frye (2) 206, (3) 136.

“ Isaac (2) 127.

“ Jacob (3) 62.

“ James (3) 136-7.

“ Rev. Rufus William (2) 149, (3) 130.

Baker, J. H. (2) 172.

“ Joseph (3) 7.

“ Rev. Osman C. (3) 125.

“ Remember (2) 22-3, 25-7, 31, (3) 4, 56.

Balcarros, Earl (2) 42.

Baldwin, Mr. (3) 70 .

“Asa (3) 64.

“ B. (2) 118 .

“ Benjamin (3) 26, 64.

“ Eleazer (2) 194-5.

“ Rev. Henry F. (3) 19.

" Rev. John (3) 35.

“ Rev. Thomas (3) 138.

" Rev. Truman (3) 51.

Ball, Benjamin (3) 195.

" Rev. Heman (2) 155, (3) 153. 
Ball, James (3) 111.

"John (3) 57.

“ Rev. Joseph (2) 203, (3) 57.

“ Levi (3) 57.

“ Peter (2) 203.

Ballard, Joseph (3) 70.

Ballou, Rev. Eli (2) 193.

"Rev. Hosea (3) 8-9.

" Rev. 1. (3) 53.

“ William S. (3) 147.

Bancroft, Harvey (3) 140.

Bangor, George (3) 152.

Banister, Mr. (3) 24.

Banker, Joseph (3) 113.

Bannister, Rev. Isaac (3) 140.

Barber, Mr. (3) 21.

" Rev. Daniel (2) 194-7, (3) 109.

“ E. D. (2) 118.

“ Elisha (3) 89.

“Job (3) 22.

“ Rev. Nelson (3) 65, 150.

" William (3) 67.

“ Rev. William N. (3) 84.

Barker, Barnabas (3) 157.

" Darius (3) 195.

“ Joseph (3) 27.

“ Nathan (3) 179.

Barlow, Hubbard (3) 69.

"William (3) 138.

Barnard, Francis (3) 7.

“ Samuel (3) 118.

Barnes, Melvin (2) 151, 167.

Barney, John (3) 80-1.

“Thomas (2) 21.

“William (3) 175.

Barnum, Barnabas (3) 161.

Barrett, Rev. B. (3) 197.

" John (3) 59, 114.

" S. (2) 222.

Barrows, Eleazer (2) 156.

Bartholomew, Eleazer (3) 45 .

Bartlett, Christopher (3) 122.

" Joshua (3) 165.

“ Rev. Robert (2) 102.

"Thomas (3) 37.

Barton, Andrew (3) 180.

"Gen. William (3) 12.

Bascom, Ira (2) 156.

" Samuel (3) 160. 
Bascom, W. F. (2) 156.

Bass, Rev. Edward (2) 195.

" Hiram (3) 27.

“ Samuel (3) 27.

Batchelder, Dr. J. P. (2) 161-2.

“ Rev. Calvin (3) 188.

“ Joseph (3) 140.

" Moulton (3) 140.

Bates, Archibald (2) 121, (3) 18.

“ Rev. Chandler (3) 126.

“ Daniel (3) 61.

“ Rev. Joshua (2) 102, 153, 155, 162.

“ Robert B. (2) 118.

Baum, Colonel (2) 45-6.

Baury, A. A. (3) 83.

" A. L. (3) 83.

Baxter, William (2) 149.

Bayley, Frye (3) 124.

"Gen. Jacob (3) 124.

“ Joshua (3) 23.

“ Samuel (3) 5.

Baylies, Nicholas (2) 124, 127, 151, 173.

Beach, Mr. (2) 139.

" Aaron (3) 54.

“ Samuel (2) 95, (3) 190.

“ Rev. Stephen (3) 69.

Beaden, Smithfield (3) 69.

Beadle, Colonel (2) 37.

Beal, Rev. Isaac (2) 102, 186, (3) 55, 136.

“ Rev. Olìver (2) 181.

“ William (3) 6.

Beaman, Joseph (2) 83.

Beardsley, Rev. Evans (3) 162.

Beedel, Colonel (3) 136.

Beck, Lewis C. (2) 162 .

Beckley, Rev. Hosea (3) 65.

Beckwith, Rev. Amos (3) 35, 169.

Beebe, Asa (3) 197.

“ Rev. Lewis (2) 127, (3) 136.

Beeman, John (3) 177.

Belknap, Dr. (2) 11. " Jesse (3) 45.

“ Simeon (2) 69.

Bell, Harvey (2) 155.

"James (3) 179.

“ Dr. John (2) 149.

“ William (2) 172, (3) 20.

Bellamy, Dr. (3) 2.

Bellow, Colonel (3) 92. 
Bellows, Ira (2) 150.

"John (3) 86.

Beman, Joseph (2) 83.

" Rev. N. S. S. (2) 155.

“ Nathan (2) 34.

Benedict, Professor (1) 19.

“F. M. (2) 149-50.

“ Prof. G. W. (1) 127, 149-50.

Bennett, Caleb (3) 119.

“ J. N. (3) 33.

“ Milo S. (2) 124.

Benson, Mr. (3) 21.

" Egbert (2) 83.

Bent, David (3) 123.

Bentley, James (3) 59 .

“ Samuel (3) 71.

“ Thomas (3) 59.

“ Rev. William (2) 185.

Benton, Samuel (3) 22, 147, 150.

Berber, Elisha (3) 89.

Berto, Benjamin (3) 90.

Bidwell, George (3) 164.

Bigelow, Major (2) 38.

“ Rev. Henry (3) 116.

“ Joel (3) 150.

“ Jonathan (3) 80.

“ Reuben (3) 138.

“ Timothy (3) 119.

“ William (3) 80, 82-3.

Billings, Elkanah (3) 68.

Bingham, Luther G. (2) 156.

" Luther L. (3) 128.

. Rev. Moore (3) 22, 68, 143.

"Solomon (2) 128.

Binney, Colonel (3) 167.

Birchard, Austin (2) 127.

Bird, Amos (3) 45 .

“ John H. (2) 150.

Bisco, Isaac (3) 4.

Bishop, Enos (3) 33.

" John (3) 117.

“ Nelson (3) 184.

“ Rev. Truman (2) 181.

Bivens, Ebenezer (3) 1.

Bixby, Samuel (3) 82.

Black, Jacob (3) 23.

Blair, Ezekiel (3) 21.

" Horace P. (2) 151.

Blake, Ephraim (3) 157. 
Blake, John (3) 29.

" Samuel (3) 158.

Blanchard, Mr. (2) 216.

"Amos (3) 108.

“ Jeremiah (3) 189.

“ Joseph (3) 58.

“ William (3) 96.

Bliss, Daniel (3) 182.

“ Rev. Enos (3) 28, 131.

“ Hosea (2) 151.

“ Joshua (3) 42.

“ Zenas (3) 98.

Blodget, Samuel (3) 59.

Blodgett, Asa (3) 59.

“ Rev. Dan (3) 71.

“ Rev. Heman M. (2) 150.

“ Rev. Luther P. (3) 73, 98.

Blood, Rev. Caleb (2) 148, 159, 185.

" Rev. Friend (3) 192.

"Isaac (3) 152.

Blythe, S. C. (2) 151.

Boardman, Mr. (3) 56 .

" Benajah (3) 91.

“ Rev. E. J. (3) 63, 146.

Bogard, Isaac H. (2) 129.

Bogue, Rev. (3) 122.

Boies, Rev. William (3) 172.

Bolster, Mr. (3) 190.

Bond, Asa (3) 52.

Bondfield, George (3) 59.

Booge, Rev. Publius V. (2) 148, (3) 73.

Boscawen, Admiral (2) 12.

Bostwick, Rev. (2) 194.

“ Gideon (3) 109.

" Samuel (3) 89.

Bovee (Bovay), Alvan Earl (2) 170.

Bowdich, Dr. (3) 17.

Bowe, Daniel (3) 138.

" Jacob (3) 138.

Bowen, Silas (2) 151.

Bowman, Bela (2) 151.

“ Rev. Francis (2) 150.

Boyce, George (3) 30.

Boyden, Major (2) 78.

Brace, Jonathan (2) 126.

Brackett, Anson (2) 151.

Braddock, General (2) 8, 206.

Bradford, Rev. Moses (3) 75. 
Bradley, Andrew (3) 69.

“ Elisha (3) 94.

“ Joshua (3) 195.

“ Peleg (3) 94.

“ Stephen R. (2) 58-9, 78-9, 83, 107, 118-19, 123, 155, 173, 223 , (3) $118,188$.

“ T. M. (3) 169 .

“ William C. (2) 29, 149, 223, (3) 188.

Bradstreet, Colonel (2) 12-13.

Brainerd, Rev. Asa (2) 151

“ Rev. Elijah (3) 146.

“ T. G. (3) 147.

Branch, Isaac (1) 176

" Rev. Thomas (2) 181.

Brant, Indian Chief (2) 67.

Bratlin, Mr. (3) 191.

Brattle, Colonel (3) 28.

Brayton, William (2) 124.

Breck, D. (3) 147.

Breckenridge, James (2) 20-1, 27, 49, (3) 14-18.

$$
\text { " Major (3) } 59 .
$$

Bresani, Father (1) 21.

Brewer, Thomas M. (1) $64,66,80,83,85-7,90-1,94,100$.

Brewster, Charles (3) 94, 171.

“ Ephraim (3) 61.

Breyman, Mr. (2) 45-6, 48.

Bridgeman, Mr. (2) 127, 204. John (3) $73,177$.

Brierly, Rev. B. (3) 164 .

Briggs, E. N. (2) 118.

“ Peter (3) 81.

“ Seth (3) 150.

Brigham, Joel (3) 7 .

" Paul (2) 118, (3) 130.

Bristed, Rev. John (2) 151.

Bristol, Mr. (3) 159.

Broadhead, Rev. John (3) 6 .

Brock, John (3) 138.

"Walter (3) 8.

Bronson, Rev. Abraham (2) 148, 150, 155, 194, 196, 197, 198-9, (3) $4,109$.

Brooks, Paschal P. (2) 151.

Brown, Mr. (3) 97.

“ Colonel (2) 47, (3) 92.

“ Major (2) 35-6.

“ Adam (3) 140.

“ Rev. Addison (3) 29.

“ Amasa (3) 199.

“ Clark (2) 172. 
Brown, David (3) 36 .

“ Edmund (3) 58 .

"Isaac (3) 66.

“ James (3) 77.

“ Jonathan (2) 69.

“ Joseph (3) 33.

" Rev. Josiah (3) 46.

“ Luke (3) 126.

“ Nathaniel (3) 197.

“ Phinehas (3) 180.

“ S. (3) 59.

“ Rev. S. D. (3) 38.

“ Sylvanus (2) 27.

" Timothy (3) 36 .

"W. G. (3) 20.

Brownel, Mrs. (2) 222.

Brownell, Chauncey (2) 150.

“ Rev. Grove L. (2) 150.

Brownson, Amos (3) 149.

“Asa (3) 149.

" El1 (2) 150.

" Gideon (3) 169.

“ Joel (2) 127, (3) 149.

“ John (2) 150.

“ Timothy (2) 65 .

“ Timothy (3) 169.

Bruce, Rev. (3) 83.

“ James (3) 40.

“ Rev. Mansfield (3) 193.

Brush, Mr. (3) 41.

" Crean (3) 188.

“ George (2) 216

Bryan, James (2) 162.

Bryant, Rev. Robert (3) 21.

Buck, Daniel (2) 118, 127, 223.

" Daniel Azro A. (2) 118-19, 223.

Buckham, Rev. James (3) 52.

Buckingham, J. P. (2) 155.

Buckland, Isaac (3) 114.

" Crispin (3) 62.

Buell, Elias (2) 127, (3) 132.

" Ephraim (3) 45.

“ Ezekiel (2) 162.

“ Mary (2) 222.

“ Ozias (2) 149.

“ Rev. Samuel (2) 150.

Bullard, A. 'T. (3) 129.

"Isaac (2) 203.

" S. A. (3) 185. 
Bullen, Rev. Joseph (2) 177, (3) 188.

Bullock, Lovell (3) 81.

"William (3) 81.

Burge, Rev. Caleb (3) 29, 79. Jeremiah (1) 176.

Burgess, Rev. Ebenezer (2) 146, 149.

Burgoyne, General (2) $38,40-4,47-8,203$, (3) $31-2,49$.

Burling, Mr. (3) 56.

" Edward (3) 94.

Burlingame, Israel (3) 184.

Burnap, Rev. U. C. (2) 173, (3) 53.

Burnham, Barnabas (3) 117.

“ David (3) 147.

“ John (2) 121, 167, (3) 116.

“ Walter (2) 167.

“William A. (2) 168.

“ Z. P. (2) 167.

Burr, Joseph (2) 153, 168.

Burrel, Henry (3) 63 .

Burrill, Andrew (3) 90.

Burroughs, Rev. Eden (2) 102.

Burrows, Rev. L. D. (3) 125.

Burt, Asahel (3) 100.

"Benjamin (2) 127.

Burton, Doctor (2) 174.

“ Rev. Asa (2) 102, 148, 155, 173, 177, (3) 171.

“ Maj. Elisha (3) 181.

“ Rev. Henry (3) 19.

“ Jacob (3) 182.

" Simon (3) 4, 135.

“ William (2) 216.

Bush, George (3) 147.

Bushnell, Rev. Jedediah (2) 155, (3) 60.

Butler, Rev. Calvin (3) 34, 158.

“ Ephraim (3) 98.

“ Ezra (2) 100, 118-19, 127, 138, 148, 186-7, 223, (3) 182.

“James D. (2) 156.

" Zebediah (3) 151.

Butterfield, Major (2) 37.

“ Rev. George (3) 193.

“ Thomas (3) 56, 79, 170.

Button, Elias (2) 69 .

Butts, Esaias (3) 86.

“ Joseph (3) 86.

Buzzell, Rev. John (2) 189.

C.

Cahoon, Rev. C. D. (3) 145.

" Daniel (3) 108. 
Cahoon, George C. (2) 150.

"William (2) 118, 223.

Calderkin, Jedediah (3) 121.

Caldwell, Joseph (2) 123.

"William (3) 104.

Call, James (3) 7.

Rev. Joseph (2) 186, (3) 44.

Calvin, Luther (3) 62.

Cameron, John (2) 138.

Camp, David M. (2) 118, 150. Rev. N. W. (3) 64.

Campbell, Captain (3) 16.

" Rev. George W. (3) 125.

“ James (2) 151.

" Solon (2) 151.

Canfield, Rev. Ezekel (2) 181, 196.

Capron, John (3) 112.

Carden, I. (1) 1.

Carey, John (1) 176.

Carigan, Patrick (2) 222, (3) 3.

Carleton, Gen. Guy (2) 35-40.

“ Maj. R. A. (3) 32.

Carpenter, Mrs. A. (2) 222.

“Asa (3) 183.

“ Benjamin (2) 118, 127, (3) 81, 83.

“ Christopher (2) 151.

“ David (3) 101.

“ Ira (3) 80.

“ Isaiah (2) 172.

“ Jonathan (3) 22.

“ Joseph (3) 96.

“ Josiah (2) 21.

“ Luther (2) 127.

“ Susanna (2) 222.

Carr, Robert (3) 136.

Cartier, James (2) 1.

Carver, John (2) 3.

Case, Loyal (2) 127.

“Lyman (3) 132.

“ M. P. (3) 26.

Cassin, Lieutenant (2) 130.

Castle, Mr. (3) 68.

" Abel (3) 68 .

Catlin, Alexander (2) 151.

" Guy (2) 149.

“ Rev. Russell (2) 196, (3) 4, 24.

Cazier, J. Le C. (2) 150, 160, 162.

" Rev. Matthias (3) 45.

Chadwick, A. G. (2) 172. 
Chaffee, Ezra (3) 5.

Chaffy, Daniel (3) 89.

Chamberlain, Abner (3) 171.

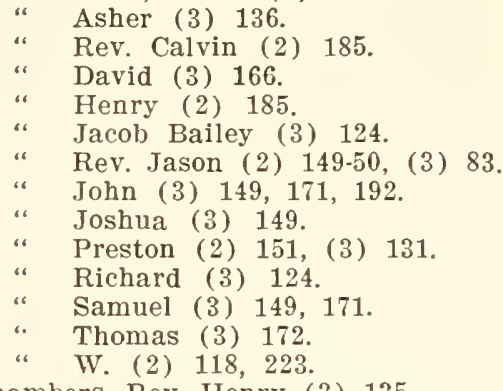

Chambers, Rev. Henry (3) 135.

Champlain. Samuel (1) $5,145,213$, (2) 2, (3) 48.

Chandler, Judge (2) 28-9.

" Rev. Amariah (2) 102, 150, (3) 178.

“ B. (2) 150.

“ John (3) 52-3, 80.

" Dr. John (1) 176.

“ Moses (2) 150.

“Thomas (2) 118, 123, (3) 52-3.

Chaney, Henry (2) 149-50.

Chapin, Lewis (3) 99.

“ Rev. Pelatiah (3) 195.

“ Rev. Sylvanus (3) 33, 133.

“ Rev. Walter (2) 155-6, 173-4, 179, 198.

“ Rev. William A. (3) 61.

Chaplin, John H. (2) 150.

Chapman, Mr. (2) 33, (3) 195.

“ Edmund (3) 40.

“ Rev. George T. (3) 38, 150.

Charlevoix, Father (1) 21, 145, 149.

Charter, John (3) 133.

Chase, A. P. (3) 185.

“ Rev. Calvin Y. (3) 59.

“ Rev. Carlton (2) 194, 199, (3) 150.

“ Dudley (2) 118, 124, 223, (3) 24, 147, 149.

“ Paul (3) 80.

“ Peter (3) 90.

“ Philander C. (2) 195, (3) 24.

" Seth (3) 24.

Chauncey, Josiah (3) 70.

Cheedle, John (3) 142.

"William (3) 7.

Cheever, Samuel (3) 93. 
Cheney, E. E. (3) 125.

"Rev. Joseph (3) 116, 158.

Chichester, Rev. Elijah (2) 181.

Child, Ebenezer (3) 102.

“ Gardner (2) 150.

“ Rev. Willard (2) 155, (3) 139.

Childs, F. (2) 150.

" Dr. Henry H. (2) 166.

Chllson, Joseph (3) 71.

Chipman, Mr. (2) 196, (3) 31.

“ D. (2) 118, 155, 173.4, 198-9, 223.

“ John (3) 113.

“ Jonathan (3) 113.

“ Nathaniel (2) 83, 120, 123-4, 127, 155, 173, (3) 172.

“ Samuel (2) 172.

Chittenden, Rev. Bethuel (2) 148, 195-6, (3) 24, 143, 161.

“ Martin (2) 95-7, 118-19, 148-9, 151, 223.

“ Noah (2) 127, (3) 43.

" Samuel (3) 175.

“ Thomas (2) 51, 54, 58-9, 62-4, 66-7, 72-4, 79, 84-8, 107, $118-19,150$, (3) $4,97,192-3$.

“ Truman (2) $149,170$.

Church, William H. (3) 97.

Churchill, Ezekiel (3) 92.

"Janna (3) 92.

“ Jesse (3) 91.

" John (3) 92.

“ Josiah (3) 92.

“ S. (3) 92 .

“ Samuel (3) 91.

“ Silas (3) 92.

"William (3) 92.

Clack, Spencer (2) 150.

Claflin, Daniel (3) 86.

“ Ebenezer (3) 86.

“ James (3) 86.

Clapp, Caleb (3) 118.

" Ebenezer (2) 150.

“ Rev. Joel (2) 149, (3) 22, 24, 118, 161, 199.

" Joshua (3) 118.

“ Reuben (3) 118

“ Rev. Rufus C. (3) 172.

Clark, Colonel (2) 80-1, 95.

“ Alonzo (2) 166.

“ Augustine (2) 118, 127.

“ Rev. Danlel A. (3) 19.

“ Ebenezer (2) 127.

“ E. L. (3) 107.

“ Ichabod G. (3) 123. 
Clark, Isaac (2) 127, (3) 96.

"J. S. (3) 122.

" Sir James (2) 48.

“ John (3) 123.

“ Joseph (3) 29.

" Rev. Kendrick (2) 102.

“ Rev. Laban (2) 181.

“ N. (2) 58-9.

"Nathan (2) 118, (3) 18.

“ Rev. Philetus (3) 173.

“ Rev. Samuel (2) 149-50, 219, (3) 29, 38, 192.

“ Satterlee (2) 150.

“ Stephen (3) 123.

“ W. (3) 111.

Cleveland, Rev. Aaron (3) 27.

“ Rev. Charles (3) 161.

“ Edward (3) 147.

“ Norman (2) 151.

“ Oliver (3) 70 .

Clinton, Gov. George (2) 52, 56-7.

“ Sir Henry (2) 65.

Cobb, Daniel (2) 170.

" Col. Ellis (3) 12.

“ James S. (2) 150.

“ S. (3) 132.

“ T. (3) 132.

Coburn, Loammi S. (3) 126.

Cochran, Robert (2) 22, 27, 32.

Cochrane, Rev. Sylvester (3) 143.

Coffein, Capt. John (3) 47.

Coit, Rev. Joseph H. (3) 4.

"William (3) 184.

Colburn, Zera (3) 40.

Colby, Ezekiel (3) 58. John C. (2) 151.

Colden, Lieut. Governor (2) 18, 32, 108.

Cole, Colonel (2) 9.

“ Rev. Joseph (2) 186.

“ Matthew (3) 149.

“ R. G. (1) 69.

“ Seth (2) 167, (3) 149.

“ Simeon (3) 157.

“ Timothy (3) 35 .

Coleman, Eliphalet B. (2) 150.

" Rev. James (2) 181.

“ Rev. Lyman (2) 155, 168.

Coley, William (2) 125.

Collamer, Jacob (2) 124, 149, 150, 166.

Collins, Mr. (2) 216. 
Collins Rev. Aaron (3) 193.

“I. (1) 1.

“ John (3) 37.

“ Rev. Samuel (3) 61.

Colt, Harris (3) 78.

Columbus, Christopher (2) 1.

Colvin, Benjamin (2) 21.

Comings, Jonas (3) 42.

Conant, Horace (2) 156.

“ Samuel (3) 96.

“ Stephen (2) 222-3.

Converse, Rev. J. K. (3) 38.

“ James (2) 151, (3) 184.

Cook, B. G. (2) 172.

“ Benjamin (2) 222, (3) 191.

“ Elkanah (3) 55.

“ Samuel (2) 74.

Cooley, Captain (2) 80-1.

“ Dr. (3) 21.

“ Benjamin (3) 139.

“ Gideon (3) 139.

Coolidge, C. (2) 118.

Coombs, Simeon (3) 97.

Cooper, Elijah (2) 150.

"Thomas (3) 194

Copeland \& Allen (2) 124.

" J. W. (2) 124.

Corbin, Pliny M. (2) 150.

Corey, John (3) 60.

Corliss, Susanna (2) 222.

Cornell, Rev. Joseph (2) 185.

Corning, Capt. M. (2) 216.

Cornwallis, General (2) 66 .

Cory, Timothy (3) 33.

Covel, Alanson L. (3) 90.

Coville, Rev. Joseph S. (3) 156.

Cowal, Joseph (3) 91.

Crafts, Ebenezer (3) 60-1.

“ Samuel C. (2) $100-1,118-19,130,148,150,223$, (3) 60.

Craig, Rev. Jesse (3) 19.

Cram, John S. (2) 170.

Crane, Rev. S. A. (3) 114.

" S. R. (3) 114.

Crawford, Rev. Joseph (2) 181.

Crome, Rev. Denzie (3) 35.

Crosby, Rev. Aaron (3) 65.

“ Rev. C. E. P. (2) 187.

Cross, Captain (3) 35.

" Shubal (3) 34. 
Crowley, Abraham O. (3) 123.

Crown, John (3) 172.

Cull, Rev. Alexander H. (3) 118.

Culver, Daniel (3) 185.

" Eliakim (3) 162.

“ Erastus D. (2) 151.

“ Rev. Lyman (3) 79, 133.

“ Nathaniel (3) 92.

" Phinias (3) 56.

" Samuel (3) 185.

Cummings, Mr. (2) 204.

Currier, Rev. John (3) 10.

Curtis, Abel (3) 104.

"Asahel (3) 104.

“ Ebenezer (2) 127.

“ Elias (2) 69.

“ Harvey (2) 156, (3) 28.

“ Israel (3) 194.

Cushman, Isaac N. (2) 170.

" J. (2) 127.

"Rev. Rufus (3) 70.

Cutler, Mr. (3) 78.

"Amos (3) 27.

" Rev. David (3) 35.

" Nathaniel (3) 60.

“ Dr. Samuel (2) 199.

“ Thomas (3) 80 .

D'Aillebout, - (2) 4.

D'Wolfe, John (2) 162.

Daly, Rev. John B. (2) 202.

Damon, Ebenezer (3) 100.

“ Rev. George (3) 198.

Dana, Isaac (3) 142.

“ John W. (2) 127, (3) 143.

" Nathan (3) 92.

“ Orlando N. (2) 162.

Daniels, Dr. (1) 23.

“ R. (3) 197.

Darling, Levi (3) 86.

Davey, Israel (2) 162.

Davidson \& Burt (2) 120.

Davis \& Porter (2) 172.

" Charles (2) 118.

“ E. (2) 172.

“ Experience (3) 147.

“ Rev. Henry (2) 102, 153, 155.

“ Rev. H. M. (3) 176. 
Davis, Dr. Ira (2) 170 .

“Jacob (3) 41-2, 119, 157.

" Rev. Joel (3) 8, 192.

“ Levi (3) 8, 27.

“ Nathaniel (3) 119.

“ Gen. Parley (3) 41-2, 119.

“ Samuel S. (2) 156.

Day, Mr. (2) 95.

Irad C. (2) 151.

Dean, Mr. (2) 95.

“ Cyrus B. (2) 121.

“ James (2) 146, 149-50.

“ Rev. Paul (3) 10.

“ R. (2) 107.

" Reuben (2) 133.

Debeline, M. (2) 7, (3) 8 .

DeLaplace, Captain (2) 34.

de Levy, M. (2) 12.

Dellins, Rev. (2) 6 .

Deming, B. F. (2) 223.

" C. (2) 127.

“ Charles F. (2) 151.

" David E. (2) 151.

“ Leonard (2) 173

“ Pennel (3) 192.

Denison, Gilbert (3) 83.

“ Henry (3) 83.

“ Henry C. (2) 170.

“ John (3) 98.

" Rev. John (2) 150.

“ Joseph A. (2) 151.

“ Rev. William C. (3) 93.

Dennett, John (2) 95.

DeRouville, M. (2) 5 .

deVaudreuil, M. (2) 7.

Devens, Rev. David S. (3) 29.

Dewey, Elijah (2) 127, (3) 17, 64.

" Rev. Jedediah (2) 20, 23, 176-7, (3) 15, 19.

“ Julius Y. (2) 151.

“ Noah (3) 25, 71.

“ Rev. Timothy (2) 181.

“ Thomas (3) 116.

DeWitt, Simeon (3) 83.

Dibble, Charles (3) 22.

Dieskare, Baron (2) 8-9.

Diller, Rev. J. W. (3) 114.

Dillingham, Mr. (2) 119.

Dike, Peter (3) 25.

Doane, David (2) 150. 
Dodge, Ebenezer (3) 112.

" John Preston Davis (3) 112.

“ Rev. John R. (3) 76.

“ Rev. N. B. (3) 175.

“ Nehemiah (2) 150.

“ Solomon (3) 119.

Donnelly, Robert (3) 193.

Donnely \& Hill (2) 172.

Doolittle, Ephraim (3) 31, 162.

“ Joel (2) 124, 127, 155-6.

Dorman, Rev. Eben H. (2) 150, (3) 69, 73, 170.

Dorril, Mr. (2) 203.

Dorwin, Lewis (2) 151.

Dougherty, Rev. James (3) 116.

Douglass, Mr. (2) 33-4.

"Jabez A. (2) 170.

“ William (3) $59,75$.

Dousman, John B. (2) 151.

Dow, Rev. J. A. (3) 125.

“ Rev. Lorenzo (2) 181, (3) 122.

Downer, A. (2) 222.

Downie, Commodore (R. N.) (2) 96 .

Drake, Rev. C. B. (3) 152.

Draper, Rev. Samuel (2) 181.

Drucaur, Chevalier (2) 12.

Drury, Rev. Amos (3) 70, 153.

Dudley, Eleazer (3) 170.

"Rev. John (3) 184.

“ Simeon (3) 114.

Duncan, George (3) 128.

"William H. (2) 170 .

Dundas, Major (2) 63.

Dunham, J. (2) 118, 172.

"Captain Josiah (3) 130.

Dunmore, Governor (2) 108.

Durfee, Mr. (3) 21.

Durkee, Bartholomew (3) 142.

“ John (3) 142, 165.

Durnford, Lieut. (2) 46.

Dustin, Daniel (3) 61.

“ Rev. Paul (2) 181.

Dutcher, James C. (2) 150.

Dutton, Rev. John (3) 143.

"Salmon (3) 48 .

Dwier, Rev. Jeremy (3) 135.

Dwight, Dr. (1) 21, (2) 152, (3) 5, 49.

"Joseph (3) 10.

Dyer, Ebenezer (3) 126.

“ Rev. Robert (2) 181.

“ Timothy (3) 110. 
E.

Earle, Joseph (3) 62.

Eastman, Mr. (3) 69, 162.

“ Calvin (3) 34 .

“ Charles G. (2) 172.

“ Cyprian (3) 34.

“ Francis S. (2) 151, 173.

“ Rev. Hezekiah (2) 185.

“ Jonathan (3) 34.

“ Rev. Tilton (2) 102, (3) 146.

Easton, Mr. (3) 17.

Eaton, Amos (2) 162.

“ Ebenezer (2) 172, (3) 63.

“ Samuel (2) 172, (3) 99.

“ Rev. William (2) 118, (3) 51.

Edgell, Rev. John Q. A. (2) 151.

Edmond, David (2) 155.

Edmunds, David (2) 127.

Edson, Rev. Ambler (3) 140.

“ Rev. Jesse (3) 85.

Egerton, Lebbeus (2) 118, 130.

Eldridge, C. G. (2) 172.

Elkins, Curtis (3) 174.

“ Henry (3) 136.

“Jonathan (2) 71, (3) 136-7.

Ellery, Benjamin (3) 161.

Elliot, Edmund (3) 91.

“ Lieut. (3) 59.

“ Israel (2) 150.

“ James (2) 118, 223, (3) 29, 83.

“ Rev. Joseph (2) 150.

Elliott, Rev. Moses (3) 147.

“ Samuel (3) 29, 83.

Ellis, Jabez (3) 23.

Elmore, Aaron (3) 112.

“ Jesse (3) 67.

“ Martin (3) 67.

" Samuel (3) 67.

Elton, Rev. Romeo (3) 195.

Ely, Rev. Richard M. (3) 53.

Emerson, Rev. Rufus (3) 188.

Emmons, Alden (2) 151.

“ Benjamin (2) 127, (3) 151.

"Solomon (3) 194.

Enos, General (2) 65-7.

" Roger (3) 67, 178.

Evans, Edward (3) 146.

“ Henry (3) 81-2.

"Simon (3) 5 . 
Evans, William (3) 146.

Evarts, James (3) 73 .

" Luther (3) 128.

" Reuben (3) 73.

Everest, Zadock (3) 1-2.

Everett, A. H. (2) 151.

" Horace (2) 149, 223.

F.

Fairbanks, E. (3) 157, 193.

$$
\text { "T. (3) } 157 .
$$

Fairchild, John (3) 147.

"William (3) 135.

Fane, Mr. (3) 126.

Farley \& Goss (2) 172.

Farnsworth, Mr. (3) 47.

“ General (2) 78 .

* \& Churchill (2) 172.

.* Rev. J. D. (2) 127, 148-9, 151, 187.

“ John (3) 132.

Farnum, Samuel (3) 172.

Farrand, Daniel (2) 118, 123, 127, 148. William (3) 73.

Farrar, Isaac B. (3) 67.

“ Rev. Joseph (3) 66.

“ Rev. Thomas (3) 65.

Fasset, Amos (3) 43, 67.

Fassett, John (2) 123-4, (3) 4, 17-18, 43-4, 69.

"Jonathan (2) 58, 64, (3) 43, 139.

Fay, Rev. Charles (3) $89,176$.

“Rev. Cyrus (2) 170.

“ David (2) 123, 127, (3) 18.

“ Dinah (3) 111.

" Rev. Don (3) 184.

“ Elijah (2) 20.

“ John (2) 149, (3) 72 .

“ Dr. Jonas (2) $24,49,51,58,64,67,71,74,123$, (3) 17.

“ Joseph (2) 63-7, 118, (3) 17-18.

“ Josiah (2) 120.

“ Samuel (3) 18.

“ Stephen (2) 24, (3) 17-18, 41.

"Theodore S. (3) 18.

Fenton, Jacob (3) 130.

Fenwick, Rev. Benedict (2) 202.

Ferguson, John (3) 164.

Ferrel, Captain (2) 10.

Ferris, Mr. (3) 71.

" Benjamin (3) 133. 
Ferris, John A. (2) 151.

" Lynde C. (2) 151.

" Peter (3) 135.

Fessenden, Joseph (2) 219.

" T. G. (2) 172.

“ William (2) 172.

Field, David (3) 80.

" Elisha (3) 14

“ Timothy (3) 188.

Fisher, Abial (2) 150.

“Amos (3) 75.

" Jonathan (3) 145.

“ Noah (3) 1.

“ Timothy (3) 1.

" W. A. (3) 20.

“ William (3) 133.

Fisk, Ebenezer (3) 177.

“ James (2) 124, 148, 150, 223.

“ Joel (3) 128.

" Jonathan (2) 21, (3) 16.

“ Josiah (2) 127.

“ Nathan (3) 128

“ Samuel (3) 85.

“ Rev. William (2) 102, (3) 6, 83.

Fitch, Gov. (Conn.) (3) 171.

" Ichabod (3) 96.

“ Jabez (3) 94-5.

“ Rev. John (2) 102, 149, 155, (3) 63, 116.

“ Leonard (2) 151.

“ Nathan (3) 81-2.

Flagg, Horatio (3) 93.

“ James M. (2) 156.

“ Dr. Theophilus (3) 93.

"William (3) 23.

Flanders, Jacob (3) 52.

Fletcher, Colonel (2) 65-6.

“ Elijah (2) 150.

“ Horace (3) 19.

“ Isaac (2) 118, 149, 151, 223.

“ James (3) 30 .

“ Jesse (3) 104.

“Josiah (3) 47, 104.

“ Samuel (3) 97, 173.

Flint, Rev. Jeremiah (3) 63.

“ Samuel (2) 150.

" Silas (3) 27.

Follett, Martin D. (3) 67.

"Timothy (2) 149-50. 
Foot, Alvan (2) 127, 149

“ Daniel (3) 113.

“ Rev, Lyman (Luman) (2) 150, (3) 4, 83, 143.

“ Nathan (3) 59.

“ Solomon (2) 150,162 .

Forbes, General (2) 13.

“ Abner (2) 155.

“ Rev. Darius (3) 53.

Forsyth, Rev. William (2) 102.

Foster, Captain (2) 37.

" Rev. (2) 102.

“ Rev. Amos (3) 145.

“ Ezekiel (2) 203.

“ Jesse H. (2) 151.

“ Joel (3) 190.

“Jonathan (3) 5 ,

“ Rev. Luke B. (2) 150.

Fowler, Rev. Bancroft (2) 155, (3) 195.

“ Barnet (3) 8 .

“ Jacob (3) 22, 23, 58.

“ Jonathan (3) 8 .

“ W. H. (2) 129.

“ Rev. William C. (2) 155.

Fox, John (2) 167.

Francis, Colonel (2) 41, (3) 91.

“ Rev. Daniel D. (2) 151, (3) 21.

Franklin, Walter (3) 126.

Frarey, Rev. Milo (3) 83.

Fraser, Captain (3) 40, 45.

" General (2) 40, 42-3, 48.

Freeman, Seth (3) 140.

“ Rev. Silas C. (2) 150.

“ Thomas (3) 7-8.

“ William (3) 7 .

French, Alvah R. (2) 127.

“ David (2) 150.

“ Elijah (3) 27.

“ Rev. Justus W. (2) 156, (3) 10.

“ Rev. L. S. (3) 132.

“ Samuel (3) 87.

“ William (2) 29.

Frizzle, Joel (3) 119.

Fry, John (3) 57.

Fuller, Rev. Cyrenus M. (3) 64 . “ Rev. Stephen (3) 177.

Fulton, Robert (2) 215.

Fyler, Roman (3) 37. 
G.

Gaffield, Benjamin (2) 68, (3) 177.

Gage, General (3) 155.

George (3) 64.

Gale, Rev. Elijah (3) 148.

“ Samuel (3) 29.

“ Rev. Solomon (3) 34.

Gallup, John (2) 151.

"Joseph A. (2) 161-2, 165-6, 173-4, 221.

Galusha, Elon (2) 150.

“Jonas (2) 93-4, 98, 118-19, 123, 127, 149, (3) 159.

Gamble, Lieut. U. S. N. (2) 96.

Gansevoort, General (2) 73.

Gardiner, Rev. (2) 199.

Gardner, Benjamin (2) 21.

Garfield, Rev. Eri (3) 24.

Garrison, William L. (2) 171.

Garvin, Ephraim (3) 94.

Gaskell, Silas (2) 173.

Gates, General (2) 38-40, 47-8.

$$
\text { "William (2) } 127 .
$$

Gay, Rev. Bunker (3) 83, 177.

Geer, Shubal (3) 3.

William (3) 3.

Gerlach, P. (2) 42.

Germain, Lord (2) 40, 43, 65, 71-2.

Gibbs, Giles (2) 69.

Gibson, Rev. William (3) 155.

Glddings, Mr. (2) 196.

Gilbert, Rev. J. (3) 187.

"Thomas (3) 47.

Gillet, Rev. Daniel C. (3) 51.

Gillett, Rev. E. (2) 151.

Gilman, Constantine (2) 150.

"Solomon (3) 112.

Gilson, Michael (3) 145, 177.

Girley, Royal (3) 83.

Glazier, Lyman (3) 96.

Glines, Jeremiah (3) 107.

Glover, Gen. John (3) 74.

Goddard, Rev. Samuel (3) 57, 130.

Godfrey, Samuel E. (2) 98, 121.

Godman, Dr. (1) 24.

Going, Rev. Jonathan (2) 149-50.

Goldsbury, John (3) 10.

Goodale, J. Harrison (3) 125.

Goodall, Rev. David (3) 85.

Goodell, Rev. Jesse (2) 177, (3) 188. 
Goodell, Shubael (2) 208.

“William (2) 156, (3) 75.

Goodenough, Ebenezer (3) 80.

Goodhue, Josiah F. (2) 156, (3) 145, 162, 193.

Goodman, Rev. Eldad W. (3) 51, 164.

Goodrich, Allen (3) 21.

“ Chauncey (2) 120, 124.

“ Hezekiah (3) 130 .

“ William (3) 22.

Goodwlllie, David (3) 9, 135.

Rev. Thomas (2) 102, (3) 9, 155.

Goodwin, John (2) 162.

Gordon, General (2) 69.

" Alexander (3) 76.

Gorham, Mr. (2) 216.

Goss, Samuel (2) 172.

Govan, Rev. Andrew (3) 9 .

Graham, C. (3) 22.

“J. A. (2) 173-4.

“ John A. (2) 195.

Grandy, Elijah (3) 32, 135.

Granger, Calvin (3) 129.

Graves, Rev. Horatio N. (3) 173.

“ Rev. Increase (3) 33.

“ Samuel (3) 186.

Greaton, Colonel (2) 28.

Greeley, Allen (2) 156.

Green, Alden (2) 121, 171.

“Asa (2) 150.

“ Rev. Beriah (3) 28.

“ Columbus (3) 56 .

“ Ebenezer (3) 131.

“ Rev. Henry (2) 148-50, 186, (3) 60, 179.

“ Dr. Horace (2) 161-2.

“ Joseph (3) 123.

Greenleaf, Rev. (3) 176.

“ Jeremiah (3) 80.

“ Stephen (3) 29.

Gregg, Lieut.-Col. (2) 45.

" John (2) 90.

Gregory, Daniel (3) 57.

Grey, Rev. Jordan (3) 22, 118, 173.

Gridley, Dr. Selah (2) 160-2.

Grigg, Rev. John (3) 4, 199.

Griswold, Mr. (3) 180.

“ Benjamin (3) 34 .

“ Bishop A. V. (2) 198-200.

“ Horace (2) 150.

“ Rev. John F. (3) 88, 126, 136. 
Griswold, R. W. (2) 172.

“William A. (2) 118, 127, 146, 148-9.

Gross, Ezra C. (2) 150.

"Thomas (2) 127, (3) 87.

Grosvenor, E. C. (2) 150.

Grout, Hilkiah (2) 68, (3) 177.

“ Jonathan (3) 107.

“ Theophilus (3) 100.

Grow, Rev. Timothy (2) 185, (3) 88.

Guernsey, Rev. H. (3) 6.

Guilder, Andrew (3) 73.

Gurley, Rev. Ebenezer (2) 177.

H.

Hackett, Rev. George (3) 174.

“ Mr. (3) 169.

Hadley, James (2) 162.

Hagar, Jonathan (2) 199.

Haldimand, General (2) 26, 63, 65-7.

Hale, Colonel (2) 42, (3) 91.

“ Abigail (3) 52.

“ Enoch (2) 151, (3) 58, 151.

“ Salma (2) 149, 151.

Hall, Captain (3) 45.

“ Lieut. (3) 45.

“ Charles (2) 167.

“ Daniel (3) 8.

“ Edwin (2) 156.

“ Eliakim (3) 185.

“ Elijah (3) 8.

“ Elisha (3) 79.

“ Enoch (3) 79, 96.

“ Frederick (1) 176, (2) 155-6.

“ Hiland (2) 223, (3) 60 .

“ Jacob (3) 8.

“ Jeremiah (3) 19.

“ I. E. (2) 151.

“ Lot (2) $123,127,155$, (3) 188.

“ Richard (2) 156.

“ Rev. Samuel R. (3) 57, 61.

“ Sarah (3) 8.

“ Thomas (3) 183.

“ William (2) 127,155 , (3) 75.

“ William F. (2) 151.

Halping, Rev. Ebenezer (3) 152.

Halsey, Silas (2) 129.

Hamilton, General (2) 40.

" Frank H. (2) 162. 
Hamilton, Jamin (2) 151.

“ John P. (2) 151.

" Silas (3) 191.

Hammond, Jonathan (3) 147.

Hampton, General (2) 95.

Hand, Rev. R. C. (3) 63.

Hapgood, Thomas (3) 147.

Hard, Rev. Anson B. (2) 196, (3) 4, 89.

Hardenbrook, J. K. (2) 151.

Harding, John (3) 104.

Hardwick, Jonas Bundy (3) 86.

Harmon, Mr. (2) 22.

" Calvin (3) 133.

“ Daniel W. (2) 173, (3) 132.

" Nathan (2) 127.

“ Reuben (2) 135, (3) 152.

Harrington, Antipas (3) 100.

“Isaac R. (2) 150.

“ Rev. William (3) 139.

“ William C. (2) 148-9.

Harris, Edward (2) 134, (3) 85, 87.

“ Jedediah Hyde (2) 127, 170.

“ Oliver R. (2) 162.

Harrison, William H. (2) 104.

Hart, Susanna (2) 222, (3) 193.

Harvey, Alexander (3) 8, 26.

" Rev. Alfred (3) 33.

" Robert (2) 127.

"Solomon (3) 5.

Harwood, Benjamin (3) 14.

“ Eleazer (3) 14, 139.

“ Peter (3) 14.

Haskell \& Palmer (2) 172.

“ Rev. Daniel (2) 146, 148, (3) 37.

“ Prince (3) 7 .

Haskins, Joseph (3) 70 .

Hassler, Mr. (1) 1.

Haswell, Anthony (2) 118, 120, 124, 127, 171-2, 222-3.

" Nathan B. (2) 216.

Hatch, Rev. James (3) 193.

" Uriel C. (2) 150.

Hathaway, Mr. (2) 66.

" Shadrack (3) 133.

" Simeon (2) 21, (3) 16.

Hathon, Anson E. (2) 151.

Haven, Augustus (3) 142.

“ Rev. Kittridge (3) 24, 93, 162.

Havens, Robert (2) 69, (3) 152, 160.

Hawkins, James (3) 119. 
Hawkins, John (3) 30.

"Joseph (2) 222 (3) 30.

Hawks, Colonel (2) 7, (3) 48.

Hawley, Elisha (3) 194.

“ Gideon (3) 71 .

“ Jehiel (2) 21, 26, 194, 196, (3) 3, 17.

"Josiah (3) 4.

Hay, Udney (2) 127.

Haynes, Asa (3) 80, 84.

“ Rev. Lemuel (3) 153.

“ Rev. Sylvanus (2) 102, 186, (3) 116.

Hazeltine, Jonathan (3) 145.

“John (3) 173.

Hazelton, Betsey (3) 124.

“ Jane (2) 222.

Hazen, General (2) 6s, (3) 105, 136-7.

“ Rev. Austen (3) 23, 87.

“ Jasper (2) 173, 190, (3) 198.

“ Richard (2) 17.

“ Rev. Wright (3) 19.

Head, Catherine (2) 222.

Heald, Amos (3) 53.

“ Daniel (3) 53.

Heath, Jonathan (3) 40.

“ Phineas (3) 22.

Heaton, Moses (3) 178.

Hebard, Rev. Ebenezer (3) 28.

" Rev. Jedediah (2) 185.

" Rev. John (2) 185.

“ William (2) 127.

Hedding, Rev. Elijah (2) 181.

Hemenway \& Sherwin (2) 172.

Hemenway, Daniel (2) 156.

Henderson, Thomas (2) 20.

Hendrick, Nathaniel (3) 114.

Herrick, Samuel (2) 45, (3) 129, 163.

“ Rev. Stephen L. (2) 151.

Herrington, Theophilus (2) 118, 123.

Hertel, M. (2) 4.

Hewes, Sophia A. (2) 174.

"W. (2) 172.

Hewett, Aaron (3) 140.

Hewlett, Mr. (2) 98, 121.

Hibbard, David (2) 127. " Ithamer (3) 93, 143.

Hick, Mr. (3) 14.

Hickok, Mr. (3) 92.

"Benjamin (3) 91. 
Hickok, David (3) 92.

“ Ellzabeth (3) 91.

“ Rev. Henry P. (2) 151.

“ Justin (3) 91.

“ Milo J. (2) 156.

“ Samuel (2) 149.

“Uriah (3) 91.

“ William C. (2) 150.

Hicks, Rev. (3) 83.

" Rev. John A. (3) 153.

“ Rev. Peleg (3) 37.

“ Whitehead (3) 152.

Higbee, Jared (3) 156.

" Lewis (3) 156.

Hill, Colonel (2) 43.

" George W. (2) 172.

“Ira (2) 150.

“ Isaac (2) 151.

“ Rev. Lewis (3) 149.

“ Nathan S. (3) 61.

“ Rodney D. (2) 151

Hilliker, John (3) 89, 170.

Hills, Mr. (3) 78 .

" I. (3) 106.

Hinckley, Mr. (3) 75 .

Hinckley \& Fish (2) 172.

Hine, Abel (3) 89.

Hinman, Rev. Clark T. (3) 125.

"Timothy (3) 63-4.

Hitchcock, Henry (2) 150.

“ Lucius (2) 151.

“ Medad (3) 186.

" Samuel (2) 120, 148-9.

Hobart, James (3) 23.

" Rev. James (3) 23.

“ Bishop (2) 200.

Hobbs, Mr. (3) 186.

Hodges, George T. (2) 130.

" Seth (3) 143.

“ Rev. Silas H. (2) 155, (3) 53.

Hodgkins, Thomas (3) 138.

Hoisington, Joab (3) 198.

Hoit, Noah (2) 162.

" Rev. William H. (2) 199, (3) 114.

Holbrook, Dr. (1) 121-4.

" John (2) 219, (3) 29.

“John C. (2) 219.

“ Levi (2) 150.

Holbrook \& Fessenden (3) 30. 
Holburne, Admiral (2) 10.

Holden, Ephraim (3) 5.

Hollyday, Noah (3) 136.

Hollister, Rev. Edward (3) 63.

Holly, James (3) 149.

" Robert (3) 34.

Holmes, Rev. H. B. (2) 203, (3) 164.

“ Rev. Samuel (3) 44.

“ Rev. William (3) 186.

Holt, Stephen (3) 138.

Holton, Isaac (2) 150.

Holyoke, Dr. (1) 22.

Hooker, Rev. Edward W. (2) 155, (3) 19.

“ Joseph (3) 171.

“ Riverius (3) 1.

“ Simeon (2) 222 .

“William G. (2) 155

Hopkins, Daniel (3) 175.

“ David (3) 107, 158.

“ Ebenezer (3) 139.

“ Bishop J. H. (1) 127, (2) 173, 199-201, (3) 38.

“ Rev. Josiah (2) 155, 173, (3) 128.

“ Roswell (2) 118, 120, 127, (3) 100.

" Samuel G. (2) 150.

Hopkinson, David (3) 79.

Horton, Lieut. (2) 69.

" Gideon (3) 27, 93.

“ Rev. William (3) 195.

Hoskins, Nathan (2) 173.

Hosmer, Mr. (3) 79. John (3) 25.

Hotchkiss, Rev. V. R. (3) 143.

Hotten, Ebenezer (3) 53.

Hough, Mr. (2) 120.

" Benjamin (2) 31.2.

“ George (2) 171-2.

“ Rev. John (2) 155-6, (3) 176.

“ Rev. Justus S. (3) 2.

Houghton, Maj. E. (3) 85. George W. (2) 150.

House, John (2) 69-70, (3) 24.

“ Nathaniel (3) 3.

“ Stephen (3) 67.

Hovey, Alvah (3) 64.

“ Daniel (3) 59.

“ Rev. Jonathan (3) 182, 189.

Howard, Mr. (3) 34.

“ Dr. (3) 21.

“ Abner (3) 171. 


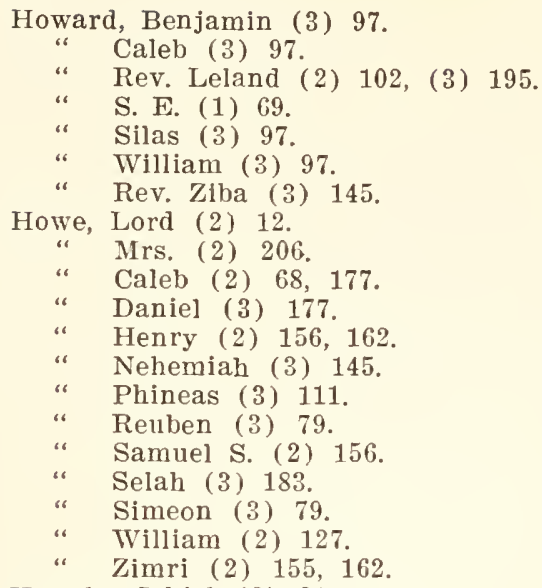

Howely, Jehiel (2) 21.

Hoxsie, Warren (2) 150.

Hoyt, Rev. Otto S. (2) 149, 155-6, (3) 90.

Hubbard, Colonel (2) 45.

“ Rev. (3) 19.

" Anson (3) 107.

“ Rev. Austin O. (3) 86.

“ Ebenezer (3) 187.

“ Eleazer (3) 23.

“ I. W. (2) 139.

“ John (2) 222.

“ Jonathan H. (2) 123, 199, 223.

“ Joseph (3) 70, 184.

"Lucius (3) 53.

“ Samuel (3) 73.

"Thomas (3) 91.

“ Rev. T. S. (3) 165.

" Timothy (2) 130.

Hubbell, Rev, Lucas (2) 149-50.

Hudson, Barzilla (3) 22.

" Charles (2) 173.

" Henry (2) 3.

Hugh, John (3) 45.

Hull, Jehiel (3) 190.

Hungerford, Samuel (3) 69 .

Hunt, Arad (2) 153, (3) 44.

“ Daniel (3) 175.

“ Elisha (3) 80.

“ Jonathan (2) $118,127,148,155,223$, (3) $29,44,72,80,177$. 
Hunt, Samuel (3) 80.

Hunter, Rev. Eli S. (3) 179.

“ Rev. H. (2) 151, (3) 55.

“ William (2) 127, 223.

Huntington \& Fitch (2) 172, 175

Huntington, Amos (3) 159.

“ Christopher (3) 151.

“ Daniel (3) 159.

" Rev. Elijah (3) 27.

“ George R. (2) 150-1.

“ Rev. J. P. (3) 187.

“ S. (2) 127.

“ Thomas (3) 151.

Huntley, Isaiah (3) 98.

"Rev. Jonathan (2) 187.

Huntoon, Dr. Ariel (1) 10.

Hurlburd, Rev. (3) 90.

" Oliver (2) 155-6.

Hurlbut, Elias (3) 96 .

"Jonathan (3) 193.

“Josiah (3) 166.

“ Rev. Salmon (3) 149.

Hutchins, Nathan (3) 129.

" Rev. S. (3) 19.

Hutchinson, Mr. (2) 119.

“ Rev. Aaron (3) 198.

“ Abijah (2) 69.

“ Edwin (2) 150.

“ Rev. Elijah (3) 195.

“ Elisha (3) 143.

“ Henry (2) 151.

“ John (2) 69.

“ Orramel (2) 151.

“ Titus (2) 104, 124, 148, 150.

Hyde, Archibald W. (2) 150.

“ Aruna W. (2) 162.

“ Azariah (3) 147.

“ Dana (3) 84.

“ Diadama (3) 94.

“ Ebenezer (3) 96.

“ Rev. Eli (3) 158.

“ Eliphalet (3) 191.

“ Jabez Perkins (3) 94.

“ Jedediah (2) 127, (3) 94-5.

“ Joshua (3) 114.

“ Major R. B. (3) 94. 
I.

Ide, Ichabod (3) 53.

“ Rev. John (2) 187, (3) 90, 132.

" Simeon (2) 120, 124.

Ingersol, Captain (2) 10, 21.

" Rev. George G. (2) 28, 102, 108, 189, (3) 38.

" Rev. John (3) 139.

Ingraham, Rev. Ira (3) 28, 133.

Irish, William (3) 116.

Isham, Joshua (3) 156.

" Oram (3) 150.

Ives, Amos (3) 123.

" Ebenezer (3) 123.

"Jonah (3) 123.

“ Rev. William (2) 202.

J.

Jackson, Abraham (3) 123, 179.

“ Andrew (2) 100.

“ Gen. Alonzo (2) 170, (3) 27, 196.

" Rev. William (2) 155, (3) 64.

Jacobs, S. (2) $11 S$.

" Stephen (2) 83, 127, 155.

" Rev. Whitman (2) 185.

James, Mr. (3) 79.

“ Dr. Edwin (1) 176.

“ John (3) 79.

Janes, H. F. (2) 118, 223.

Jarvis, William (3) 184.

Jefferson, Thomas (2) 90-2.

Jennie, Rev. Prince (3) 140.

Jennison, Silas Hemminway (2) 102-4, 118-9, 170.

Jenny, Lyman T. (2) 151.

Jennings, Benjamin (3) 60 .

"James (3) 42.

Jerrells, Thomas (3) 181.

Jewett, Mr. (2) 124.

" Luther (2) 127, 172, 223, (3) 125.

Joe, Captain (Indian) (2) 206, (3) 95, 123.

Johns, Benjamin (3) 55.

" Jehiel (3) 94.

Johnson, Mr. (3) 47.

“ General (2) 11.

“ Rev. (3) 20.

“ Colonel (2) 8-10, 47, 71, (3) 137.

" Captive (3) 47.

“ David (2) 205.

" Eden (3) 34.

" Edward (3) 52.

“ Edwin Ferry (2) 170, (3) 54, 110. 
Johnson, Isaiah (3) 52.

" J. (1) 19.

“ Rev. James (3) 157, 193.

“ John (2) 206-7, (3) 25, 68, 89, 184.

“ John H. (3) 114.

“ Lewis (2) 150.

“ Moses (3) 1.

"Rev. Noah (3) 140.

“ Thomas (3) 124, 137.

“ Wakeman G. (3) 90.

“ Sir William (2) 12, 15.

"William S. (3) 99.

Jones, Abner (2) 190-1.

" Alexander H. (2) 151.

“ Asa (3) 30.

“ Darius (2) 172.

“ Rev. David A. (3) 63.

" E. (2) 216.

“ Henry (2) 218.

“John (3) 169.

“ Rev. Jonathan (3) 147-8.

" Reuben (2) 51, 58, (3) 57.

Joslin, Josiah (3) 100.

Judd, E. W. (3) 114.

June, David (3) 27.

Justin, Benjamin (3) 191.

K.

Kane, Edward (2) 151.

Keeler, Mr. (3) 91.

" Aaron (3) 67, 94.

Keith, Mr. (3) 55.

" Caleb (2) 170.

“ Rev. Reuel (2) 156.

Kellogg, Mr. (3) 45, 91.

“ Daniel (2) 127, 149.

“ Rev. E. W. (3) 73, 98.

“ Rev. Gardner (3) 26.

“ Horace (3) 164.

" Orson (2) 150.

"Sherman (3) 93, 133

Kelly, John (2) 13, (3) 104, 152, 174.

“ Rev. S. (3) 125.

Kemp, Mr. (2) 21.

Kendall, Mr. (3) 5 .

B. F. (2) 172 .

Kendrick, Rev. Clark (3) 143.

Rev. N. (2) 150 .

Kennan, Jairus (2) 146, 149-50. 
Kenney, Rev. (3) 20.

"Thomas (2) 75.

Kennedy, John (3) 25.

Robert (3) 25.

Kent, Rev. Brainerd (3) 90.

“" Caphas (3) 64.

“ Rev. Dan (2) 102, (3) 21.

“ Elisha (3) 152.

“Jacob (3) 124.

“ John (2) 69.

Kenyon, Jared (2) 150.

Keyes, Danforth (3) 86.

“ Elias (2) 223, (3) 165.

“ James (3) 3.

" Stephen (3) 40.

Kidder, Rev. Corbin (3) 29.

“ Rev. Thomas (3) 195.

Kilburn, Rev. Alanson (3) 68 .

"John (2) 150.

Kimball, Asa (3) 12.

“ David M. (3) 29.

" George (3) 47.

“ Dr. Gilman (2) 166.

“ Rev. James (3) 173.

“ John (3) 120.

“ Rev. Moses (3) 146.

King, Dauphin (2) 150.

" N. T. (3) 129.

" Reuben (3) 77.

“ Salmon (3) 78

“ Samuel (3) 110-11.

Kingsbury, Major (3) 59.

" Ebenezer (3) 98 .

" Rev. Samuel (2) 186, (3) 97.

Kingsley, Rev. Phineas (3) 175.

Kingston, Major (2) 48.

Kinney, Bradford (3) 140.

" Rev. Jonathan (3) 140.

Kinsley, Stephen (3) 43.

Kitchell, Harvey D. (1) 156.

Knapen, Rev. Mason (3) 90, 133, 168.

Knapp \& Jewett (2) 120, 124.

" Abraham (3) 96.

“ C. I. (2) $118,172$.

Kneeland, Joseph (2) 69.

Knickerbacker, Mr. (3) 68.

John (2) 107.

Knight, Horatio (2) 222.

“ Nathaniel (3) 79.

“ Samuel (2) 123, 127, (3) 29. 
Knight, Simeon (3) 150.

Knights, Capt. (2) 78-9.

Knockburn, (Surveyor) (2) 24-5.

Knowles, Sir Charles (2) 7.

Knowlton, Calvin (3) 126.

" Luke (2) 123, (3) 7, 126.

L.

Labaree, Mr. (3) 47.

" Rev. Benjamin (2) 153, 155.

“ Joseph (3) 98.

Lacy, Isaac (3) 64.

Ladd, Rev. David M. (3) 68.

Lafayette, General (2) 99, 147.

Lamb, Mr. (3) 83.

"Aaron (3) 31.

“ Rev. Dana (2) 150-1, (3) 33.

“ Edward (2) 167.

“Jonathan (2) 173.

“ Rev. Silas (3) 186.

Lambert, Rev. Amos B. (2) 155.

“ Rev. Nathaniel (2) 102, (3) 125.

Lamphire, Mr. (3) 191.

Lane, Freeman (3) 109.

“ Hannah (3) 165

“ Jedediah (3) 98.

“ Samuel (3) 37.

Langdon, Benjamin F. (2) 162.

“ Chauncey (2) 155, 161, 223.

“ M. G. (2) 162.

" Rev. Solomon (2) 181.

Lansing, John (2) 83.

Latherbee, Benjamin (3) 75 .

Lathrop, Joshua (3) 52.

" Leonard E. (2) 162.

Law, Mr. (3) 78.

"Rev. Solomon (3) 53.

Lawrence, General (2) 12.

“ Rev. Byram (2) 151.

“ Isaac (3) 89.

“ Stephen (3) 37.

Lawson, Rev. Samuel (3) 195.

Lawton, Rev. John (3) 193.

Leach, Rev. G. (3) 20.

"Jonathan (3) 110.

Learned, General (3) 19.

Leavens, Jedediah (3) 147.

Leavenworth, Henry (2) 150.

“ Jesse (3) 40, 62.

“ Nathan (3) 90. 
Leavitt, Rev. (Hervey) Henry F. (2) 155, (3) 176.

" Seth (3) 181.

Lee, Colonel (2) 80.

" Rev. Chauncey (3) 37, 169.

“ Rev. Jesse (2) 181.

"Rev. Jonathan (3) 189.

“ Noah (3) 45

Leech, Joseph (3) 67.

Leland, Rev. (3) 83.

" Rev. Aaron (2) 118, 155, 186-7, (3) 53.

“ Luther (3) 64.

Leonard, David (3) 133.

" Rev. George (2) 102, (3) 24, 114, 195.

“ Seth (2) 173-4.

Lester, Charles G. (2) 150.

LeSueur, M. (1) 135.

Leverett, John (2) 127.

T. (2) 118 .

Levings, Noah (2) 173.

Lewa (Indian) (2) 206.

Lewis, Abner (3) 55.

"Jonathan (3) 100.

“ Lyman (2) 170.

Lideus, Colonel (3) 55.

Lincoln, General (2) 47.

“ Benjamin (2) 150.

Linsley, Joel (3) 60.

“ Rev. Joel H. (2) 150.

Lindsey, Rev. John (2) 102.

Livingston, Major (2) 35.

Logan, Mr. (3) 160.

London, C. I. M. S. (3) 53.

" Lord (2) 10.

Long, Clement (3) 147.

Loomis, Rev. Aretas (3) 20.

“ Beriah (2) 127.

“ Rev. Jacob N. (3) 86.

“ Luther (2) 146, 149, 207.

“ Warren (2) 149-50.

Lord, Joseph (3) 157.

" Samuel (3) 181.

Lough, J. (2) 216.

Loveland, Aaron (2) 170.

“ Rev. Samuel C. (2) 173-4, 192, (3) 147.

Lovell, Rev. Alexander (3) 176.

Lovewell, Captain (2) 205.

"Nehemiah (3) 59, 137.

Low, Mr. (3) 56.

Lowd, Allen (2) 129.

Luce, Elibu (2) 138. 
Luce, Jonathan (3) 59.

Lull, Captain (2) 80.

" Timothy (3) 88.

Lunt, Rev. (3) 184.

Lydius, Henry H. (2) 26.

Lyman, General (2) 8.

“ Ebenezer (3) 139.

“ Rev. Elijah (2) 102, (3) 35.

“ Rev. Gershom C. (2) 155, (3) 110.

“ John (3) 34.

“ Joseph (3) 110.

“ Josiah (3) 192.

“ Phineas (3) 34.

“ Rev. Solomon (3) 143.

Lynde, Cornelius (3) 192.

Lynds, Daniel (3) 80.

Lyon, Asa (2) 149, 223, (3) 77 .

" Dan (2) 216.

“ James (3) 174.

“ Matthew (2) 134, 172, 195, 223, (3) 70.

“ Samuel H. (2) 151.

Mc.

McClair, Mr. (3) 56.

McClintock, James (2) 161-2.

McCallom, Rev. Jacob S. (3) 53.

McClure, Mr. (3) 140.

McConnel, Mr. (3) 99.

" Stevens (3) 26.

McCormick, James (3) 193.

McCrea, Jane (3) 50.

Mccullen, William (3) 96.

McDaniel, John (3) 94-5, 122, 133.

McDonald, Rev. Lewis (3) 22, 118.

McDuffee, John (3) 26, 58.

McElroy, Mrs. (3) 115.

McEwen, George (3) 89.

McFairlan, Jesse (3) 149.

McGaffey, Andrew (3) 50.

McGee, Rev. Jonathan (3) 29.

McGinnis, Captain (2) 9, 10.

McIntosh, Donald (3) 176.

McKeen, David (3) 59.

"Rev. Silas (3) 26.

McKeith, Thomas (3) 172.

McKillips, Rev. G. M. (3) 73 .

McLachlin, Archibald (3) 136.

McLaughlin, Mr. (3) 111.

McNeal, John (3) 171. 
McNeil, John (3) 51.

McNaughton, Alexander, (2) 22.

M.

Macdonough, Commodore (2) 95-7, (3) 50, 176.

Maclean, Colonel (2) 36.

Macomb, General (2) 96-7, 145.

Macrae, W. F. (1) 175-6.

Madison, James (2) 93.

Maeck, Jacob (2) 150.

Magoon, Alexander (3) 63.

Malada, William (3) 33.

Malary, R. C. (2) 223.

Mallary, Odden (3) 185.

"Rollin C. (2) 155 .

Manley, John (3) 64.

Mann, Charles (3) 52.

“ Rachel (3) 193.

" Robert (3) 172.

" Rev. W. M. (3) 125.

Manning, Rev. Abel (3) 140.

" Rev. Joel (3) 3.

Mansfield, Amos (3) 116.

March, Alden (2) 162.

Marsh, President (U. V. M.) (3) 87.

“ Abel (3) 146.

"Amos (2) 148.

“ Anna (2) 219.

“Asa (2) 121.

“ Charles (2) 127, 223.

“ Rev. Daniel (3) 19.

" Isaac (3) 112.

" George P. (2) 173.

“ James (2) 147-8, 150, (3) 87, 182.

“ Joel (3) 24, 160.

“ Joseph (2) 118, 127, 150, (3) 87, 132, 146.

“ Perley (2) 219.

" William B. (3) 89

Marshall, Mr. (3) 190.

Martin, Mr. (2) 127.

“ Caleb (3) 34

“ George (3) 138, 199.

“ Joseph (3) 141 .

“ Peter (3) 94.

“ Rev. Solon (3) 57, 59.

Martindale, Rev. Stephen (3) 172, 179.

Marvin, Ebenezer (2) 127, (3) 73.

Mason, Daniel (3) 60 .

"John (2) 54. 
Mason, Joshua (3) 199.

“ Peter (2) 69.

“ Rev. Reuben (3) 183, 187.

“ Rev. Samuel (3) 150.

Mather, Cotton (3) 111.

" Dan (3) 111.

“ Dr. William (2) 162, (3) 110.

Matthews, Aaron (3) 116.

“ Darius (2) 155.

“ Joel (3) 128.

“ Thomas P. (2) 161-2.

Mattison, Rev. Isaiah (3) 159.

"Thomas (3) 159.

Mattocks, John (2) 124, 223.

" Samuel (2) 118, 127.

Mattoon, Abel (3) 6.

May, James (3) 12.

Mauxa Wuxal (Indian) (2) 206.

Maxham, E. (2) 172 .

Maxwell, Mr. (3) 85.

Meacham, Hine (3) 89.

“Jacob (3) 89.

" James (2) 156, (3) 21, 128.

“ John (2) 162, (3) 70.

Mead, Rev. Enoch (3) 128.

“ James (2) 32.

“ Stephen (3) 109, 139.

"Thomas (3) 115.

Mears, Rev. Roswell (3) 73.

Meech, Mr. (2) 100, 119.

"Ezra (2) 130, 149, 223.

Meeker, Rev. B. O. (3) 34.

“ Eli (3) 179.

Melgs, Elizabeth (2) 86.

Melvin, Captain (2) 68.

Mendall, Amos (3) 30.

“ Lucy (3) 30.

Merrifield, Mills (3) 60 .

Merrill \& Langdon (2) 172.

Merrill, F. F. (2) 118.

“ John (3) 36.

" O. C. (2) 223.

“ Selah (2) 162.

“ T. (2) 118.

“ Rev. Thomas A. (2) 155-6, 176, 179, (3) 114.

Merriman, Charles P. (3) 125.

Merritt, John (3) 99.

Mervin, Ebenezer (2) 195.

“ S. J. M. (2) 168.

Messenger, Mr. (3) 97-8. 
Messer, Rev. Asa (2) 150, (3) 139.

Miles, H. H. (2) 167.

Miller, Jonathan P. (2) 170.

“ Rev. M. D. (3) 194.

“ Samuel (2) 153, 155, (3) 70.

Milligan, Rev. James (3) 155.

Millnes, Sir Robert S. (2) 90.

Mills, Samuel (2) 175.

Miner, A. L. (2) 118.

" Burrill S. (2) 151.

" Rev. Lawson (3) 60.

“ Ovid (2) 172.

Minkler, Ezekiel P. (2) 151.

Mitchell, (Indian) (2) 206.

" C. L. (2) 162.

" Rev. Joseph (2) 181.

“ Rev. William (2) 149, (3) 153.

Molly, (Indian) (2) 206, (3) 95.

Monckton, Colonel (2) 8.

Monroe, Colonel (2) 11.

" President James (2) 97-8, (3) 167.

“ N. W. (3) 176.

Monta, Joseph (2) 222.

Montague, Samuel (3) 14, 43.

Montcalm, General (2) 10, 11, 14.

Montgomery, General (2) 31, 34-6, 47.

Moody, Rev. Eli (3) 189.

Moore, Elisha (2) 150.

" Fairbank (3) 29.

“ Rev. Ferris (3) 145.

" George (2) 146.

“ Sir Harry (3) 25.

“ Henry (3) 58.

“Isaac (2) 150 .

“ James (3) 25.

“ John (3) 5, 140.

" John W. (2) 172.

“ Jonah (3) 35.

“ Paul (3) 162.

“ Samuel (3) 52.

"Thomas (3) 52.

“ Thomas Porter (3) 52.

Moredock, Thomas (3) 52.

Morey, Samuel (3) 71.

Morgan, Mr. (2) 95.

“ Rev. Asaph (2) 150, (3) 68.

“ Rev. Stillman (3) 59, 172.

"Thomas (3) 116.

“ Willam (2) 100-1.

Morley, John (2) 150. 
Morrill, Abraham (3) 63, 189.
“ G. L. (2) 151.
“ Justin S. (3) 167.
" Sargeant (3) 63.

Morris, Rev. Henry (3) 133.

" Henry Villiers (2) 170.

“ Lewis R. (2) 83, 118, 223, (3) 163.

Morrison, Robert (2) 80.

Morse, Mr. (3) 79.

“ David (2) 22, (3) 182.

“ John H. (1) 26, (3) 182.

“ Rev. Josiah (3) 157.

“ Moses (3) 122.

“ Percival (2) 151.

Morss, Rev. (3) 195.

Morton \& Willard (2) 172.

Morton, Rev. Daniel O. (2) 155, (3) 162, 164.

Mosely, Increase (2) 118, 123, 127.

Mott, Willis (2) 127.

Moulton, Samuel (2) 162.

Mower, Naham (2) 172.

Mudge, John (3) 140.

“ William (3) 140.

Munro, John (2) 21-3, (3) 16.

Munson, Caleb (3) 182.

" John (3) 169.

Murdock, Rev. James (2) 146, 149.

“ Thomas (3) 130.

Murray, Eber (3) 133.

“ Lord (2) 12, 16.

“ Orson S. (2) 172, 218.

N.

Nash, Rev. Sylvester (3) 156.

“ Timothy (3) 79.

Neal, Benjamin (3) 2.

Neel, Hughbartes (3) 58.

Nelson, Captain (3) 59.

“ Mr. (3) 191.

“ Robert (2) 162.

Newell, General (2) 97.

“ George (2) 150.

" Gordon (2) 127.

“ Timothy (3) 60.

Newman. Rev. Davld (3) 177.

Newton, C. G. (2) 151.

“ Ephraim H. (3) 110.

“ John (3) 7. 
Newton, Marshall (3) 162.

Nichols, Colonel (2) 45.

“ Rev. Ammi (3) 27.

“ Dorcas (2) 222, (3) 27.

“ G. W. (2) 172 .

“ Isaac (3) 27.

“ Rev. James (2) 4, 194-6, (3) 4, 6, 109.

" Rev. Jonathan (3) 6.

Nightingale, Joseph (3) 33.

Niles, Henry (3) 85.

" Nathaniel (2) 118, 123, 127, 223.

Noble, Mr. (3) 21.

" Rev. C. D. (3) 164.

“ Rev. Calvin (3) 52.

“ O. (2) 127.

“ Obadiah (3) 174.

“William (2) 150, 170.

Norris, Mr. (3) 86.

“ Nathaniel (3) 86.

“ Samuel (3) 58.

Norton, William (3) 94.

Nott, Rev. (3) 20.

Noyes, David P. (2) 170.

“ John (2) 127, 223, (3) 29, 83.

“ Oliver (3) 94.

Nutting, David (3) 22.

" John (3) 58.

“ Rufus (2) 173, (3) 147.

“William (2) 127,149, (3) 147.

Nye, David (3) 23.

“ Elijah (3) 23.

“ Rev. Jonathan (2) 150, (3) 126, 156.

“Thomas (2) 150 .

O.

O'Callaghan, Rev. Jeremiah (2) 173, 201-2.

O'Kelly, Rev. James (2) 190.

Oakes, Seth (3) 5.

“ William (1) 173.

Obear, Rev. Josiah (3) 22, 118.

Ogden, Rev. John C. (2) 195, 196, (3) 24.

Olcott, Peter (2) 118, 123, (3) 130.

Olds, Mr. (3) 122.

“ Rev. Gamaliel S. (2) 149, 155.

“ Jesse (3) 186, 192.

Olin, Gideon (2) 87, 118, 223.

Henry (2) 83, 118.

Olmsted, James (3) 67.

"Lewis (2) 208. 
Olmsted, Moses (3) 139.

" Seth (3) 67.

Ordway, Moses (3) 174.

Ormsbee, Benjamin (3) 35. Jonathan (2) 121.

Ormsby, Gideon (3) 109.

Osborn, Benjamin (2) 173, (3) 172, 179.

Osgood, Nahum (2) 118, 150.

Osmore, Mr. (3) 79.

Owen, Daniel (3) 186.

“ Edward (3) 139.

“ Leonard (3) 116.

P.

Packard, Rev. (3) 83.

" Rev. Winslow (3) 193.

Paddock, Ephraim (2) 124, 127.

“ James A. (2) 150, (3) 61 .

“ Robert (3) 9, 10.

“ William (2) 149.

Page, Mr. (3) 79.

" David (3) 79, 107.

“ Jacob (2) 71, (3) 137.

“ John (3) 113.

“ Joseph (3) 189.

“ Luther (3) 186.

“ Phineas (3) 100.

“ Porter (3) 86.

“ Rev. Robert (3) 86.

“ Samuel (3) 79.

"William (2) 155.

Paige, Nathaniel (3) 7 .

Paine, Charles (2) 104, 118-19, 149.

“ Elijah (2) 10, 83, 123-4, 127, 145, 151, 155, 223, (3) 128, 192.

“ Elisha (2) 73, 118, 123, (3) 128.

“Ezra (3) 131.

“ John (3) 192.

“ Thomas Morrs (3) 131.

Painter, Gamaliel (2) 153, 155, (3) 113.

Palmer, Rev. Aaron (2) 150, (3) 10.

" Benjamin R. (2) 166.

“ David (2) 165-6.

“ Rev. John E. (3) 10.

“Thomas (3) 25.

“ William A. (2) 100-1, 118-19, 124, 150.

Pangborn, John (3) 135.

Pardee, Rev. Amos (2) 196, (3) 143.

Park, Jonathan (3) 126. 
Parker, Colonel (2) 10.

" Rev. C. C. (3) 199.

“ Judge (1) 40.

"Amos A. (2) 150 .

" Rev. B. E. C. (2) 166.

“ D. (3) 123.

“ Rev. Daniel (2) 151.

“ David (3) 95.

" George H. (2) 151.

“ Rev. James (2) 185, 187, (3) 67, 175, 177.

“ Jeremiah (3) 102.

“ Joshua (3) 145.

" Ralph (3) 74 .

"Thomas H. (3) 66.

"William H. (2) 156.

Parkhurst, Jabez (2) 150.

"Joseph (3) 152.

Parks, John (3) 146.

Parmlee, Hezekiah (3) 187.

" Rev. Moses (3) 57.

“ Quartus (2) 150.

“ Randolph (3) 146.

“ Rev. Reuben (3) 89.

“ Rotus (2) 151.

" Rev. Simeon (2) 149, (3) 187, 193.

Parsons, Rev, Justin (3) 138, 165, 190.

" Rev. Silas (3) 168.

Partridge, Capt. Alden (2) 150, 169-70, (3) 100, 109-10.

" Cyrus (2) 170.

Patch, Mr. (3) 168.

Patrick, Joseph (3) 77.

Patterson, Colonel (2) 56 . " James (3) 103.

Patton, Robert B. (2) 156.

Payne, John (3) 24.

" Jonathan (3) 34.

Noah (3) 34.

Peabody, Josiah (3) 110.

Peak, Mrs. (2) 121.

"John (2) 185.

“ Samuel (3) 24.

Pearl, Colonel (2) 80-1.

Pearsall, Thomas (3) 26.

Pearson, Rev. Jacob (3) 83.

Pease, Calvin (3) 120.

“ Eli (3) 3.

"Jacob (3) 3.

Peaslee, George (2) 150.

"Zacheus (3) 40. 
Peck, George H. (1) 62.

“ Rev. John (3) 149.

“ Lucius B. (2) 103, 120.

“ Nathaniel (3) 119.

“ Rev. Phineas (2) 102.

“ Rev. Richard (3) 118.

"Thomas (3) 4, 171.

“ Rev. Thomas K. (2) 150.

Peckham, Samuel (3) 73.

Pember, Thomas (2) 69.

Peninan, Udney H. (3) 62.

Penniman, Jabez (2) 149.

Percival, Orin (3) 50.

Perham, Isaac (3) 5 .

"Jonathan (3) 5, 6 .

Perkins, Mr. (3) 52, 185.

"E. B. (2) 170 .

“ Jesse (3) 179.

“ Joseph (2) 162, 167.

“ Nathaniel (3) 179.

“ Theodore (3) 140.

“ Rev. William S. (2) 102, (3) 4.

Perrin, Zachariah (3) 23.

Perry, Mr. (3) 16.

“ Rev. Clark (3) 125.

" Rev. James (3) 140.

“ John (3) 145.

“ Rev. Josiah (3) 28.

Perrson, Rev. Ira (3) 53.

Persons, Rev. Cornelius G. (3) 3.

Petingill, E. (3) 107.

Peters, Rev. Absalom (2) 155, (3) 19.

" Andrew (3) 177.

“ Rev. Hugh (3) 4.

“ John (3) 25.

“ Rev. Samuel (1) 4, (2) 194-5.

Petibone, J. S. (3) 109.

Phelps, Charles (2) 150, (3) 111.

" Edward E. (2) 150.

" Rev. Elnathan (2) 185, (3) 133.

“James T. (3) 57.

“J John (2) 127, 150.

“ Noah (2) 33.

“ Samuel S. (2) 124, 127, 223.

" Timothy (3) 111.

Phillps, General (2) 40, 47.

“Elijah (2) 216.

“ Paul D. (3) 63.

“ William P. (2) 216.

Phipps, William (2) 5, (3) 145. 
Plerce, Benjamin (3) 105.

" Jonathan (3) 34-5.

“ Jotham (3) 132.

“ Warner (3) 164.

Pierpont, Robert (2) 103, 118, 120, 149.

Pierson, Moses (3) 161.

"Thomas (3) 161.

Plke, Mr. (3) 191.

Pingry, Nathanlel (3) 123.

Pitkin, Calvin (3) 112.

" Martin (3) 112.

“ Nathaniel (3) 112.

“ Stephen (3) 112.

Pitman, Benjamin H. (3) 110, 145.

Pitt, William (2) 12.

Pixley, Rev. Benton (3) 192.

Platt, James K. (2) 149.

Plumb, Rev. Elijah W. (2) 155, (3) 136.

Plummer, Mr. (3) 31.

Plympton, Rev. Oliver (3) 138.

Poland, Joseph (2) 172.

Pomeroy, Cassius F. (2) 150.

“John (2) 148-50.

“ John N. (2) 149-50, 207.

“ Rev. Rufus (3) 158.

“ Thomas M. (2) 172.

Porter, Captain (2) 10.

" Arthur L. (2) 149.

“ Experience (2) 156.

“ Thomas (2) 118, 123, 134, (3) 52, 172.

“ Rev. William A. (2) 149.

Post, Martin (2) 118.

"Truman M. (2) 156.

Potter, Andrew (3) 156.

" Rev. Lyman (2) 102, 177-8, (3) 130.

Pottier, Mr. (3) 160.

Powel, General (2) 40, 47.

“ Major (2) 95-6.

Powell, Felix (3) 64, 139.

“H. (2) 151.

“ T. (2) 150.

Powers, Rev. Grant (2) 205, (3) 130.

" Rev. Peter (2) 102, 124, 177.

“ Stephen (3) 198.

Pratt, Abijah (3) 7, 192.

" James O. (3) 54.

“ Joel (2) 83, 127.

“John A. (2) 127, 166.

" Rev. John B. (3) 83.

" Samuel (3) 14. 
Pratt, Timothy (3) 14.

Prentiss, Samuel (2) 124, 149, 223. Thomas (3) 184.

Prescott, General (2) 36.

“ General Robert (2) 90.

Preston, Major (2) 36.

“ Rev. Nathaniel O. (3) 19.

“ Rev. Willard (2) 146-9, (3) 38, 156.

Prevost, Governor (2) 96.

Prideaux, General (2) 13, 15.

Priest, Moses (3) 140.

Prime, Rev. Nathaniel (2) 155.

Prindle, Mr. (3) 32.

“ Rev. Cyrus (2) 180, (3) 19.

Pringle, Captain (2) 39.

Rev. William (3) 155.

Proctor, Rev. Hadley (2) 155.

Purdy, Benjamin (2) 21.

" Edward C. (2) 172.

“ Rev. Lucius M. (2) 155, (3) 143.

Proudfit, Rev. John (2) 168.

Putnam, Major (2) 10-12.

“ A. K. (3) 176.

“ Aaron (3) 75.

“ Edward (3) 75 .

“ Israel (3) 50 .

“ John L. (2) 170.

“ Seth (3) 115, 119

$\mathrm{R}$.

Randall, Rev. Benjamin (2) 188.

Phineas (2) 150.

Ranney, W. R. (2) 118, 168.

Ranny, Samuel (3) 34.

Ranslow, Rev. George W. (3) 73 .

Ransom, Dr. (3) 21.

" Rev. Elisha (2) 185.

“ Rev. John (3) 30, 93, 190.

“ Royal M. (2) 150.

“ Rev. William B. (3) 60 .

Rasier, Joseph (3) 5.

Rasler, Father (2) 6.

Ratenburgh, Thomas V. (3) 164.

Rathburn, Rev. David (3) 190.

Rawson, Leonard (2) 156.

Rev. Nathaniel (3) 86.

Raymond, Benjamin (3) 31 .

Read, Elias (3) 59. 
Read, Nathaniel (2) 150.

" Peter (3) 105.

“ Simeon (3) 104.

Redfield, Dr. (2) 167.

“Isaac F. (2) 124, 155.

Redding, David (2) 121, (3) 18.

Reed, Colonel (2) 24-6.

" David (1) 25, (2) 149.

“ Hezekiah H. (2) 127.

“ Joseph (2) 127.

“ Dr. M. M. (1) 176.

" Micah (3) 5.

“ Samuel (1) 10.

Reese, David M. (2) 162.

Reeve, Rev. Abner (2) 177, (3) 29, 111.

Remington, Rev. Jairus (3) 145.

" Zadock (3) 45.

Remmele, Samuel H. (3) 191.

Reynolds, John (3) 167.

“ Rev. T. (3) 69.

" Thomas C. (3) 3 .

Rhodes, Holden (2) 156.

Rice, Mr. (3) 85.

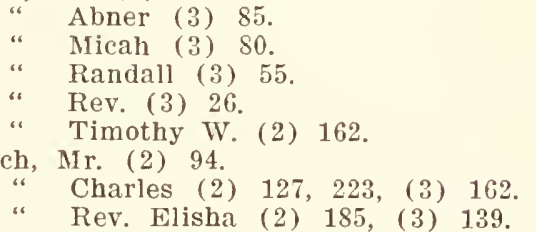

Richards \& Co. (2) 172.

“ Mr. (3) 31.

“ Rev. John (3) 198.

“ Mark (2) 118, 127, 223, (3) 188.

Richardson, Mr. (3) 31.

“Amos (3) 128.

“ Dr. (1) 25-6, 30, 35-6, 39, 61, 64, 66, 75, 86, 90, 103, 105, $141,143$.

" David (2) 170.

“ I. P. (2) 150.

“ Nathaniel (3) 128.

" Stanton (3) 128.

Rickey, Robert (2) 188.

Ridlan, John (3) 199.

Riedesel, Countess (3) 50.

“ General (2) 40, 42-3, 47.

Riley, J. (2) 124.

Robbins, Dr. James W. (1) 174-6. 
Robbins, R. D. C. (2) 156.

"William (3) 177.

“ Zenas (3) 86.

Roberts, Mr. (2) 216.

“ Eli (3) 31.

Robertson, Rev. J. J. (2) 149.

Robins, Aaron (3) 27.

“ George (3) 27.

Robinson, Amos (3) 87, 128, 131.

“ Beverly (2) 62-3.

“ Ebenezer (2) 20.

“ Eber (3) 91.

“ Elijah (2) 127.

“ Ezekiel (3) 128.

“ G. (3) 12 .

“ J. L. (3) 12.

“ James F. (2) 151.

"Jonathan (2) 123, (3) 17.

“ Joseph (2) 20.

“ Leonard (3) 14.

“ Lewis (3) 148.

“ Moses (2) 58, 64, 74, 86, 118, 123, 127, 223, (3) 14, 17, 179.

“ Nathan (3) 166.

“ Samuel (2) 19-20, 185, (3) 14, 16-18, 41-2.

“ Silas (2) 21, (3) 16 .

“ Stephen (2) 127.

Rockwell, Joseph (3) 116.

“ William H. (2) 219.

Rogers, Major (2) 14-15, (3) 8, 179.

" Jabez (2) 222.

“ James (3) 103.

“ Martha A. (3) 26.

“ Samuel (3) 9.

“ Timothy (3) 71 .

“ Rev. William (3) 148.

Ralph, Moses (2) 150.

Rood, Mr. (3) 97.

" Heman (2) 156.

Root, Abraham (3) 174.

“ Banajah (2) 177, (3) 153.

“ Eliakin (3) 149.

“ Erastus (2) 150.

“ Timothy (3) 81 .

Rosbrook, Eleazer (3) 79.

“ James (3) 79.

Rose, Justus (3) 36.

“ Samuel (3) 109.

“ \& McNeil (2) 216.

Ross, H. H. (2) 150. 
Rossetter, Mr. (3) 172.

Roundy, John (3) 150.

Rowell, John (3) 75.

Rowley, Thomas (3) 62 .

Royce, Rev. Andrew (2) 173, (3) 10, 192.

“ Elihu M. (3) 22.

" Stephen (2) 124, (3) 22.

Ruggles, Colonel (2) 9.

Rumsey, Nathan (3) 92-3.

Runnels, Major (2) 66.

Russell, David (2) 148-9, 171-2.

“ John L. (2) 151.

“Joseph P. (2) 150.

" Noadiah (3) 47.

“ Thomas (3) 69.

" Rev. Thomas (3) 24.

“ William P. (2) 162.

Ryan, Rev. Henry (2) 181.

Ryther, W. E. (2) 172.

S.

St. Clair, General (2) 40-3, (3) 91.

St. Leger, Colonel (2) 47, 65-6.

Sabin, Mr. (3) 86.

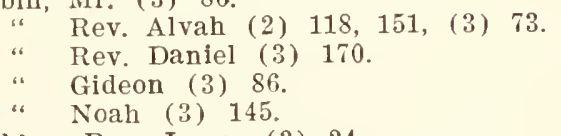

Sabine, Rev. James (3) 24.

"John T. (3) 89, 109.

Sackett, Joseph (3) 175.

Safford, Mr. (3) 86 .

"Captain (3) 24, 122.

“ David (3) 43-4.

“ Rev. Hiram (3) 38.

“ John (3) 44.

“ Joseph (3) 14.

“ Mayhew (2) 150.

“ Major Samuel (2) 20, 45-6, 64, (3) 18.

“ Silas (2) 198, (3) 70, 114.

Sage, Rev. Sylvester (2) 102, 155, (3) 188.

Salisbury, Rev. William (3) 178.

Sampson, Charles (2) 216.

" Rev. Guy C. (3) 116.

Sanborn, Ebenezer (3) 22-3.

" Rev. Eber (3) 172.

“ John (3) 136.

“ Lewis (2) 151. 
Sanborn, Rev. William (3) 172.

Sanders, Rev. Daniel C. (2) 145, 148-9, (3) 37, 176. " David (3) 73.

“ Ebenezer (3) 73.

Sanderson, James (3) 198.

Sanford, John (3) 189.

Sanhoop (Indian Chief) (3) 32.

Sargeant, Colonel (2) 71, (3) 111.

“ David (3) 29.

“ Jabez (3) 52.

“John (3) 29, 59.

“ Leonard (2) 127

“ Silas (3) 45.

“ Thomas (3) 29.

Saunders, Dr. (1) 10.

“ Rev. (3) 179

“ Admiral (2) 14.

Savage, James (3) 184.

Sandawda (Indian) (3) 191.

Sawyer, Abraham (3) 126.

“ Rev. Ephrain (2) 185, (3) 133.

" Lieut. F. A. (2) 216.

“ Frederick A. (2) 150.

" Gamaliel (2) 150.

“ Rev. Isaac (2) 187.

" James (3) 23.

“ James L. (2) 150.

“ Jesse (2) 32.

“ Joseph (3) 147.

“ Rev. Joseph (2) 102, 187, (3) 92, 159, 190

“ N. P. (3) 94.

“ Truman (3) 94

Sax, John (3) 89.

Saxton, Mr. (3) 159.

“ Frederick (3) 37.

" Jonathan (3) 71.

Sayles, Rev. Ezekiel H. (3) 69.

Scales, Rev, Jacob (3) 60 .

“ Rev. William (3) 108, 149.

Schuyler, General (2) 34-5, 40, 43-5, 47, (3) 50.

" Colonel (2) 4-5.

Scott, Aaron (3) 59.

" Rev. E. J. (3) 125.

“ Lieut. J. B. (2) 129.

“ Rev. John (3) 67.

“ Joseph (2) 127, (3) 60.

“ Moses (2) 21, (3) 16.

“ Oliver (3) 14, 152.

“ Samuel (3) 14. 
Scott, Waitstill (3) 1.

“Walter (2) 222, (3) 170.

“ William (3) 78 .

Scovel, Ezra (2) 151.

Sealy, Captain (3) 59. Abner (3) 177.

Searle, Henry (3) 38.

"Isaac (3) 91.

“ Rev. John (3) 152.

"William (3) 4.

Seaver, Comfort (3) 152.

Seavey, Joseph (3) 50.

Seleck, John (3) 91.

Sergeant, Rev. Nahum (3) 147.

Sessions, John (2) 127.

Severance, Charles C. (2) 151.

$$
\text { “ C. H. (2) } 172 .
$$

Seymour, Mr. (2) 119.

“ Epaphri (2) 219.

“ Henry (3) 83.

“ Horatio (2) 155, 223.

“ Uriah (3) 36.

Shafter, James (3) 5 .

Shaw, Daniel L. (2) 151.

" George B. (2) 124, 150.

“ Isaiah (3) 30 .

“ Samuel (2) 138, 223.

“ Rev. Samuel B. (3) 83.

Sheldon, Cephas (3) 30.

“ Elisha (3) 161.

“ Samuel B. (3) 161.

Shepard, Aaron (3) 78.

"Ashbel (3) 60, 78.

" Guy B. (2) 151.

“ Rev. Samuel (2) 155.

Shepardson, John (2) 123, (3) 80-1, 83.

" Samuel (3) 83.

Shepherd, Z. R. (2) 155.

Sherburn, Henry (3) 81.

Sherburne, Major (2) 37.

Sherman, Asaph (3) 10.

"Captain J. (2) 215-16.

“ Jonathan (3) 9 .

“ Capt. R. W. (2) 215-16.

Sherrill, Franklin (2) 156.

Sherwood, Mr. (3) 92.

" Captain (3) 91.

“ Capt. J. (2) 63.

Shields, James Van Allen (2) 170. 
Shinkley, Thomas (3) 32.

Shipman, Rev. A. (3) 172.

Shirley, Mr. (2) 8. " Governor (2) 8.

Shumway, Rev. (3) 75.

Shute \& Smith (2) 172.

Shuttleworth, Rev. Samuel (3) 195.

Sibley, Derrick (2) 171.

Sill, Rev. Elijah (3) 64.

Silloway, Hezeliah (3) 23.

Simons, Alvan (3) 189.

" John (2) 155, (3) 3.

Skeel, John (3) 136.

" Rev. Thomas (2) 102.

“ Thomas (2) 185, (3) 96, 158.

Skeene, Philip (2) 20, 28. " Major (2) 33-4.

Skelton, Rev. Thomas (3) 67.

Skinner, Richard (2) 98-9, 118-19, 124, 155, 223, (3) 109.

" Samuel (3) 35.

“ Rev. Warlen (2) 102, (3) 53.

Slade, William (2) 118, 120, 171, 173, 174, 223.

Slafter, John (3) 130.

Slason, Rev. J. I. (3) 138.

Slawson, Eleazer (3) 113

Sleeper, Samuel (3) 25, 124.

Sloan, John (3) 131.

Smalley, Benjamin (3) 113, $15 \mathrm{~s}$.

Zerah (3) 113.

Smead, Benjamin (2) 172.

Smiley, Rev. Robinson (3) 164.

" Nathan (2) 104, 119.

Smith, Mr. (2) 33, 119, (3) 31, 68.

" Addison (2) 150.

“ Rev. Albert (2) 156.

“ Benjamin (3) 24.

“ Rev. Benjamin B. (2) 200, (3) 114.

“ Bull W. (3) 120.

“ Caleb (3) 161.

" Calvin (3) 9 .

“ Chauncey (3) 21.

“ Earle (2) 150.

“ Ebenezer (3) 130

“ Rev. Elias (2) 151, 191.

" Rev. Elihu (2) 162, (3) 45, 110.

“ Elizabeth (3) 139.

" Enos (3) 52.

“ Rev. Ethan (2) 162, (3) 143.

“ Francis P. (3) 79 . 


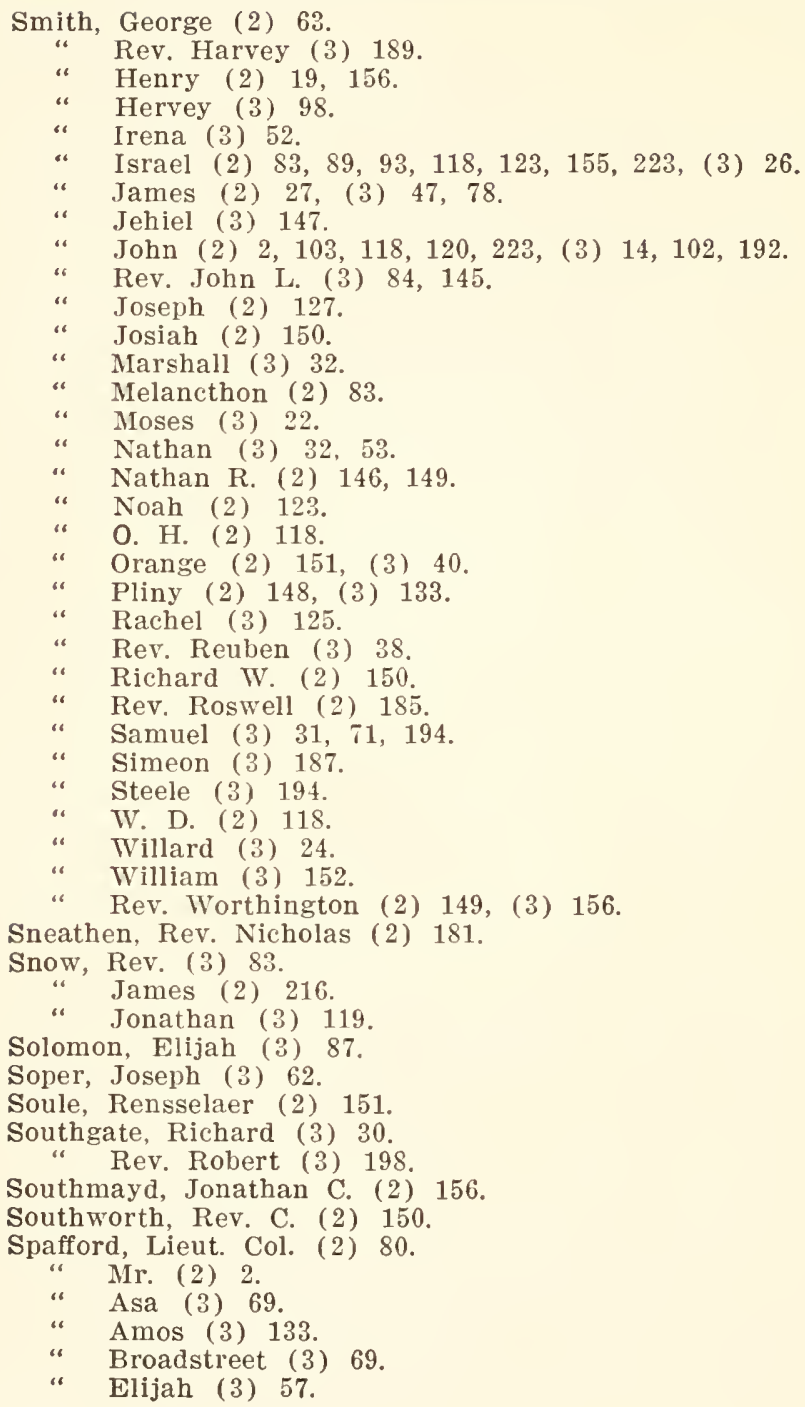


Spafford, H. G. (2) 150.

“ John (3) 43.

“ Jonathan (3) 192-3.

" Nathan (3) 69.

Sparhawk, Rev. Samuel (3) 138.

Spaulding, Mr. (3) 76 .

“ Benjamin (3) 160 .

“ J. (2) 118, 167.

“ Rev. Justin (3) 145.

“ Dr. Phineas (2) 166.

“ Philip (3) 97.

“ Timothy (3) 135.

“ William (3) 91.

Spear, Andrew (3) 147.

“ Jacob (3) 27.

“ Samuel (3) 27.

Specht, General (2) 40.

Spencer, Abel (2) 118.

" Chester (2) 162.

“ Gideon (3) 112.

“ Hubbard (3) 45.

" Seth (3) 36.

Sperry, Mr. (2) 95.

"Anson J. (2) 198.

Spicer, Rev. Tobias (2) 102.

Spooner, State Printer (2) 120.

“\& Green (2) 134.

“ A. (2) 222.

“ Alden B. (2) 150 .

“ Bigford (3) 149.

“ Eliakim (3) 152.

“ Judah P. (2) 134, 171.

“ Jeduthan (2) 124, 172.

“ Paul (2) 58, 74, 118, 123, (3) 86.

“ Wymall (2) 172.

Sprague, Mrs. (2) 222.

“ Alvah (2) 151.

“ Rev. N. (3) 152.

" Rev. William B. (2) 155.

Spring, Samuel (3) 75 .

Squier, Truman (2) 195-6.

Squire, Alde (3) 135.

Stacey, H. B. (2) 172.

Stanley, Joseph (3) 78.

"Timothy (3) 42, 78 .

Stansbury, Lieut. (U. S. N.) (2) 96.

Stanton, Joshua (2) 148.

Stanwix, General (2) 13.

Staples, Rev. O. H. (3) 195. 
Stark, Rev. Jedediah L. (3) 29.

Starkweather, John (2) 44-7, 52, (3) 48.

Starr, Peter (2) 127, (2) 127. on, David (3) 3

Stearns, Reva (3) 56, 198.

“ Ebenezer (3) 117

“John (3) 117.

Stebbins, Benjamin (2) 80.

" Ebenezer (3) 59.

Stedman, A. (3) 174.

" Nathaniel (3) 126.

Steele, Elisha (3) 89.

“ George (3) 89.

“ Rev. Joseph (3) 45.

“Joslah (3) 89 ,

“ Robert (2) 150.

“ Samuel (3) 184.

“ Zadock (3) 146.

Stevens, General (2) 69.

“ Mr. (3) 11, 158.

“ Beriah (2) 173-4.

" Clark (3) 119

“ Elias (3) 152.

“ Enos (3) 7-8.

“ John (2) 156, (3) 33.

“ Phineas (2) 7.

“ Roger (3) 139.

“ Samuel (2) 15, (3) 8.

“ Simeon (3) 163.

“ Simon (3) 84 .

“ Willard (3) 8 .

Stewart, Mr. (2) 220.

“ Rev. (2) 198.

“ Ira (2) 155.

“ John (2) 20, (3) 29.

“ Rufus (3) 122.

“ Samuel (3) 34 .

Stickney, Colonel (2) 45.

Stiles, Dr. (3) 88, 188.

“ Ezra (3) 161, 188.

“ Rev. S. (3) 21.

Stinson, Robert (3) 25.

Stockwell, Abel (3) 110-11.

$$
\text { “ Perez (3) } 111 .
$$

Stoddard, Captain (2) 10.

“ David F. (2) 156.

“ John (3) 97. 
Stoddard, Solomon (2) 155.

Stone, Mr. (3) 32.

“ Rev. Barton W. (2) 191.

“ David (3) 24.

“ Davis (2) 150.

“ E. (3) 41.

“ Rev. George (2) 151.

“ Rev. Isaiah (2) 185.

“ J. A. B. (2) 156.

“ J. F. (3) 183.

" Joel (2) 151.

“ Rev. John L. (2) 200.

“ Moses (3) 41-2.

“ Nathan (3) 126, 195.

“ Philip (3) 31.

Storer, Dr. (1) 116, 124, 135, 147-8.

Storey, Amos (3) 158.

Storrs, Aaron (3) 146.

“ John (2) 151.

“ Seth (2) 155.

Story, Frederlck (2) 167.

" William (3) 7.

Stow, David (3) 189

“"Joshua (2) 170.

Stowell, Elliot (3) 81.

“ Hezekiah (3) 81.

“ Israel (3) 147.

Streeter, Benjamin (3) 57.

Strong, Mr. (2) 119.

“ General (2) 97.

“ Aaron (3) 23.

“ Barnabas (3) 24.

“ Benajah (3) 87.

“ Elijah (2) 127, (3) 87.

“ Rev. Henry B. (2) 150, 155, (3) 156.

“ James (2) 150.

“ Joel (2) 150.

“ John (3) 1, 2.

“ Noah (3) 27.

" William (2) 127, 223.

Sturdifit, Mr. (3) 32-3.

Styles, Ezra (2) 134.

Sullivan, General (2) 37-8.

Sumner, Rev. Clement (2) 177, (3) 171. “ David H. (3) 88.

Sunderland, Peleg (2) 27, 32.

Swan, Benjamin (2) 118, 151.

Swanzey, Rev. John (3) 50.

Sweatt, Dr. William (2) 170. 
Sweet, David (3) 103.

Sweetser, Dr. William (2) 149.

Swett, Rev. Josiah (3) 196.

Swift, Benjamin (2) 155, 223.

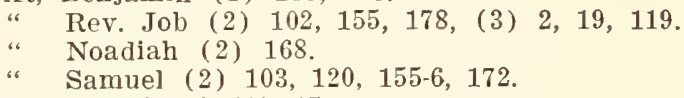

Symonds, Colonel (2) 45.

$\mathrm{T}$.

Tabor, Lemuel (3) 172.

Taft, Daniel (3) 199.

Taggard, James (3) 119.

Talcott, George (2) 129.

Taplin, Mr. (3) 58.

“ Colonel (3) 4.

“ John (3) 23, 125.

Tappan, Rev. James (3) 4.

Tarbox, James (2) 127.

Taylor, Absalom (3) 116.

“ Betsey (3) 169.

“ Eldad (3) 169.

“ Rev. Hezekiah (2) 177, (3) 126.

“ John (3) 126.

“ Kezziah (3) 169.

“ Rev. Preston (3) 178, 188.

Teasdale, Rev. Thomas (3) 19.

Temple, Robert (2) 127, 130.

Templeton, Rev. J. (3) 125.

"John (3) 119.

Ten Broek, Abraham (2) 20.

TenEyck, Mr. (2) 2.

Tenney, Samuel G. (3) 108, 178.

Thatcher, Adocia (3) 193.

" Rev. Joseph (3) 10, 174.

Thayer, Elisha (3) 186.

Thomas, General (2) 36-7.

" Rev. C. A. (2) 185, (3) 28.

" H. J. (2) 172.

Thompson, General (2) 37.

“ Abel (3) 71, 149.

“ Amos (2) 127.

“ Daniel P. (2) 120, 173, (3) 158.

“ David (3) 23, 71.

" Hezekiah (3) 194

“ Rev. Ignatius (3) 143.

“ Rev. James (3) 143.

“ Rev. John (3) 168. 
Thompson, John C. (2) 124.

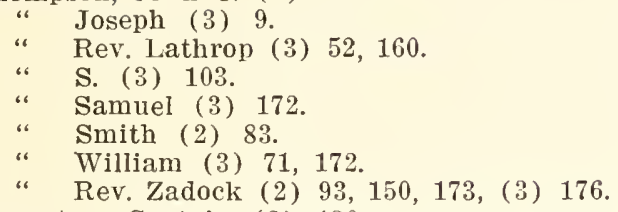

Thornton, Captain (2) 130.

Throop, John (2) 123, (3) 143, 181.

Thurber, Joseph (3) 22-3.

Thurston, Rev. Pearson (3) 157.

Tiarks, Mr. (1) 1.

Tibbits, George (2) 83.

Tichenor, Isaac (2) $74,83,88,89-93,98-9,118,123,127,223$, (3) $18,89$.

Tilden, Rev. Lucius L. (2) 155, (3) 153.

" Stephen (3) 87.

Tinker, James (2) 168.

Tinkham, Jeremiah (3) 5 .

Tisdale, Rev. James (3) 79.

Titcomb, Colonel (2) 9.

Tobey, Rev. Alvan (3) 193.

Tolford \& Fletcher (2) 172.

Tolman, Rev. Thomas (2) 120, (3) 60.

“ Mr. (3) 78.

Toomalek, - (2) 206.

Topliff, Mr. (3) 30.

Torrey, Dr. (1) 174.

" Rev. Joseph (2) 149, (3) 152.

Tousey, Lazarus (2) 151.

Towle, Brackett (3) 58.

Town, Edmund (3) 69.

Towner, Mr. (3) 31.

“ Rev, James (2) 150.

Townshend, Rev. M. (2) 151.

"Micah (2) 118,127, (3) $28,83$.

Tracy, E. C. (2) 172, 179.

" Rev. Joseph (3) 186.

Treadway, Rev. James (3) 184.

Troup, Joseph (3) 59.

Trowbridge, William (3) 91.

Truaire, Rev. John (3) 44.

Trumbull, Robert (3) 60.

Tryon, Governor (2) 22-7, 73, 108, (3) 17.

" William (3) 195.

Tubbs, Simeon (3) 37.

Tucker, J. (3) 41. 
Tucker, Rev. Joshua (3) 19.

Tuckerman, Edward (1) 175-6.

Tullar, Rev. Martin (2) 155, 178, (3) 152, 195.

Tulley, William (2) 161-2.

Tupper, Absalom (3) 71.

“ Sergeant (2) 65-6.

“ Rev. Samuel H. (2) 150.

“ Zuriel (3) 71.

Turner, Bates (2) 124, 127.

“ Bela (3) 52 .

“ Edward (2) 155-6.

Turrill, Miner Y. (2) 151.

Tuthill, Daniel (3) 101.

Tuttle, Alexander T. (2) 151.

“Amos (3) 69, 86.

“ Benoni (3) 184.

“ Hezekiah (3) 89.

“ J. W. (2) 172.

Twilight, Rev. A. L. (3) 36 .

Twining, A. C. (1) 19, (2) 155.

“ Rev. A. T. (3) 176.

Twing, Joshua (3) 11.

Twiss, Samuel (3) 41-2.

Tyler, Colonel (3) 147.

“ John (2) 104.

“ Joseph (2) 33, (3) 173.

“ Royal (2) 123-4, 148-50, 173-4, (3) 29, 8 .

“ Timothy (2) 150 .

Tyson, Isaac (3) 141 .

U.

Underhill, Abraham (3) 64.

Underwood, Amos (3) 57.

Upham, James (3) 118.

“ Rev. William D. (3) 173.

Utley, William (3) 101.

V.

Vail, Joshua Y. (2) 127, 150, 219.

" Micah (3) 62.

Vance, James (3) 74 .

Van Ness, Cornelius P. (2) 99, 100, 118, 119, 124, 151. James (2) 151.

Vannest, Rev. Peter (2) 181.

Verplank, Gulian (2) 83.

Vincent, Thomas (3) 140. 
Vinton, Lieut. D. H. (2) 129.

"Rev. John (3) 147.

Virginia, - (2) 121.

Vose, Rev. Gilman (3) 165.

Wadleigh, Rev. Frederick A. (3) 83 .

Waggoner, John (3) 89.

Wainwright, G. G. (2) 151.

Wait, Col. (3) 58.

“ Benjamin (2) 80, (3) 178.

“ Joseph (3) 36.

“ Luther (2) 150.

" Lynde (3) 71.

“ Nathaniel (3) 36.

Walbridge, Col. (2) 65, 66, 73.

“ Ebenezer (2) 127, (3) 18, 71.

Walden, J. (3) 156.

Waldo, Rev. Nathan (3) 192.

Wales, Eleazer (3) 130.

“ G. E. (2) 118, 151, 223.

“ Nathaniel (3) 96.

“ Roger (3) 52.

Walker, C. (2) 179.

" Rev. Charles (2) 102, 151, 155, (3) 29, 153.

“ Gideon (3) 190 .

“ Horatio (3) 104.

“ Jacob (3) 122.

“ Levi (3) 191.

“ S. B. (3) 93.

Wall, William (3) 124.

Wallace, Jonathan (3) 98.

“ Dr. William C. (2) 162.

Wallage, Rev. Elijah (3) 150.

Waller, C. C. (2) 172.

" David (2) 69.

Walles, Ebenezer (3) 4.

Walter, Ira (3) 36.

" Lemuel (3) $36,37$.

Walton, E. P. (2) 127.

“ John (3) 119.

Ward, - (3) 1, 31, 32, 33, 58, 71.

“ Lieut. Charles (2) 129.

“ David (2) 151.

" John (3) 32, 185.

" Col. William (3) 126, 143.

Wardner, A. (2) 118. 
Ware, Rev. Avery (3) 118.

Warner, Col. (3) 91, 92.

“ Allen (2) 130.

“ Almon (2) 150.

“ Rev. Daniel (3) 68, 182.

“ Gideon (2) 22, 32 .

“ Isaac (2) 153 .

“ Moses (3) 3, 59.

“ Seth (2) $22,23,25,26,27,30,31,32,34,36,42,44,45,46$, 51, (3) $17,32,49,66,171$.

“ Stephen N. (2) 170

“ William (3) 53.

Warren, Rev. Lester (3) 192.

Washburn, Rev. Azel (3) 152.

“ Cyrus (3) 177.

“ Daniel (3) 190.

“ Isaiah (3) 162.

“ P. T. (1) 176.

“ R. H. (3) 141.

“ Royal (2) 150.

“ Stephen (3) 109

Washburne, Rev. Cephas (2) 150.

"Rev. Ebenezer (2) 181.

“ Abisha (3) 114.

Washington, George (2) 8, 72, 73, 84, 90.

" Lieut. J. M. (2) 129.

Waterbury, Gen. (2) 39.

Waterhouse, Abel (3) 158.

“ Dr. Henry S. (2) 149, 151.

Waters, Constable (2) 78.

" E. (3) 41.

" Ebenezer (3) 27.

" John (3) 35

Watrous, Sophia (2) 173.

Watson, David (2) 172.

Watts, Dr. Robert, Jr. (2) 166.

Wead, Rev. Ira M. (2) 151.

Weare, - (2) 53, 54.

Weaver, Rev, John M. (3) 19.

Webb, General (2) 10, 11.

“ Derrick (3) 51.

" Rev. Isaac (2) 186, (3) 27.

“ Joshua (3) 150.

Webster, — (2) 220, (3) 78.

“ Abdial (3) 91.

“ Benjamin (3) 40 .

“ John S. (2) 151.

Weeks, Rev. Holland (3) 139. 
Welch, Elijah (3) 81, 84.

Weld, John (3) 147.

Wellers, Eliakim (2) 26.

Wellman, Rev. James (2) 177, (3) 188.

Wellmore, Rev. Isaac (3) 35.

Wells, Capt. (3) 45.

" Chester (3) 148.

“ Dr. Henry (3) 29.

" Hubbel (3) 85.

“ Jonathan (3) 148.

“ Col. Samuel (3) 29, 132.

“ Mrs. Susanna (2) 22, (3) 193.

“ Rev. William (3) 29.

“ William W. (2) 151.

Wentworth, Gov. Benning (2) 17, 18, 108, 196, 224, (3) 13, 58, 126.

"Moses (3) 66.

West, Ebenezer (3) 177.

"Francis (3) 41.

Weston, - (2) 124.

“ Edmund (2) 150.

John (2) 151.

Wetmore, Seth (2) 149.

Wheeler, B. I. (3) 119.

" G. (2) 150

" J. B. (3) 119.

“ Rev. John (2) 147, 148, 149, (3) 195.

“ Joseph (3) 69.

“ Justus P. (2) 150.

“ Tolman (2) 151.

Wheelock, Abijah (3) 41, 42.

"Asa (3) 41.

" Ebenezer (3) 190.

“ Dr. Eleazer (2) 144.

“ Rev. James R. (3) 10.

“ Rev. John (2) 196, (3) 189.

“ Lucinda (3) 42.

“ $\quad$ Peter (3) 41, 42

“ President (3) 171.

“ Rev. Stephen M. (2) 150.

Whelan, John (3) 27.

Whelpley, James (3) 93. Rev. S. W. (2) 150.

Whipple, Commodore (3) 50.

" Abraham (3) 50.

“ Benjamin (3) 183

“ Gen. William (2) 44.

Whitcher, David (3) 63.

Whitcomb, Lieut. (2) 69. 
Whitcomb, Asa (3) 7, 8, 165.

“ Cyrus, Jr. (3) 35.

“ Lot (3) 7.

“ Rev. Luke (3) 173.

White, Ebenezer (3) 124.

" Joel (3) 71.

" John (2) 127.

“ Jude (3) 100.

" L. S. (2) 216.

“ Mary (3) 175.

“ Noah (3) 124.

“ Phineas (2) 155, 223.

" Robert (2) 215.

" Thaddeus (3) 182.

“ Col. Thomas (3) 145.

" Thomas W. (3) 8.

“ William (2) 78 .

Whitefield, Rev. George (2) 188.

Whitelaw, Gen. J. (1) 19, (3) 50.

“ James (3) 26, 140, 154, 184.

Whiting, E. B. (2) 172.

“ Col. John (3) 190.

"Rev. Samuel (2) 177, 178, (3) 53, 150.

Whitlock, Rev. John (2) 196, 199.

Whitman, Rev. Jacobs (2) 185.

Whitmore, Gen. (2) 12.

" Francis (3) 110.

“Thomas (3) 110.

Whitney, James (3) 104, 111.

“ Jonas (3) 111.

“ Lemuel (3) 29.

“ Nathaniel (3) 111.

“ Richard (2) 118, 120, (3) 83.

Wicker, Lemuel H. (2) 150.

Wickham, Rev. Joseph D. (2) 155, 168.

Wickwire, Joseph (3) 14.

Wilcox, David (3) 88 .

" Hazzard (3) 16.

" Jacob (3) 123.

" James (2) 33.

“ Nathan (3) 122.

“ Samuel (3) $86,138$.

Wild, Rev. Daniel (3) 35.

Wilden, Rev. John C. (3) 67.

Wilkins, Rev. C. R. (3) 19.

Wilkinson, Gen. (2) 96 .

Willard, - (3) 68 .

“ Dubartis (3) 68. 
Willard, John (2) 127, (3) 107.

“ Jonathan (3) 135.

“ Josiah (3) 145.

“ Nathan (3) 29.

“ Lieut. W. T. (2) 129.

William III, King (2) 197.

Williams, Capt. (3) 45.

“ Col. (2) 9, (3) 50.

“ Maj. (2) 48.

“ Rev. A. (3) 53.

“Azarias (2) 147.

“ Charles K. (2) 5, 124, 155, 162.

“ Elijah (3) 80 .

“ George P. (2) 151.

“ Rev. Henry (3) 83.

“ Rev. John (2) 5, (3) 192.

“ Joseph (3) 131, 190.

“ Norman (2) 103, 118, 120, 150, 166.

“ Rev. S. P. (3) 147.

“ Dr. Samuel (1) 1, 2, 10, 19, 22, 29, 31, 40, 45, 50, 98, 100, 117,120, (2) $5,30,102,173,174,197$, (3) 37.

“ Rev. Stephen (3) 172.

“ William (3) 9, 13, 44, 111, 159.

Williamson, Joseph (2) 150.

Willis, Rev. (3) 9, 83.

" Samuel (3) 192.

Williston, David H. (3) 174.

“ E. B. (2) 150 .

“ Rev. Ralph (2) 181.

"Thomas (2) 132.

Willson, Jared (2) 150.

Wilmot, Rev. Ezra (3) 149.

Wilson, — (3) 83, 115.

" Lieut. U. S. A. (2) 208.

“ Boyd H. (2) 151.

“ Ebenezer (3) 133.

“ Rev. George (3) 195.

“ James (3) 26.

“ John (3) 190.

“ Rev. Jonathan (3) 145.

“ Joseph (3) 149.

“ Rev. Samuel (3) 6.

“ William (2) 151, (3) 142.

“ Rev. W. B. (3) 195.

Winans, John (2) 215, 216.

Winch, Rev. Joel (3) 129.

Winchel, - (3) 68 .

Winchester, Hubbard H. (2) 170. 
WVing, David, Jr. (2) 118, (3) 119.

Winslow, Colonel (2) 8 .

" Jedediah (3) 27.

“ Dr. Thomas (2) 170.

Winthrop, John (2) 5.

Wiswell, Henry (3) 190.

Witherill, J. (2) 223.

Witherspoon, Dr. (2) 57.

Withington, G. R. M. (2) 151.

Wolfe, Gen. (2) 12, 13, 14.

Wood, Mr. (3) 78.

“ Rev. Abner (2) 181.

“ Ebenezer (3) 119.

“ Eleazer (3) 14.

“Enos (3) 129.

“ Rev. Jacob (3) 125.

“Job (2) 126.

“ John (2) 222.

“ Samuel (2) 216 .

"Solomon (3) 129 .

"Theoda (2) 126.

" Rev. Thomas H. (3) 85.

Woodard, Samuel (3) 85.

Woodbridge, Col. (2) 47.

" E. D. (2) $128,149$.

" Enoch (2) 123, 148, 149, (3) 175.

Woodburn, John (3) 193.

Woodbury, Jesse (3) 57.

"Jonathan (3) 57.

Woodman, Rev. Jonathan (2) 102.

Woods, Rev. Abel (3) 162.

Woodward, B. (2) 118.

“ Bezaleel (2) 64, 65, 71, 123.

“ Henry (2) 150.

" Rev. James (3) 36, 130.

“ Jehiel (3) 146.

“ Rev. John H. (3) 187.

“ Dr. Jonathan D. (2) 162.

“ Joshua (3) 139, 161.

“ Rev. Nehemiah (3) 30.

“ Dr. Theodore (2) 160, 161, 162.

Woodworth, Mrs. (3) 52.

" Rev. Francis C. (3) 70.

" Ziba (3) 119.

Wool, Gen. (2) 103.

Woolage (Wollage), Rev. Elijah (3) 44, 83.

Woolcut, Elijah (3) 51.

Wooster, Rev. Benjamin (3) 60, 69. 
Wooster, Dorastus (2) 196, 199.

Worcester, Rev. John H. (3) 157.

“ Rev. Leonard (2) 148, 149 , (3) 137.

“ Rev. Samuel A. (2) 150, (3) 147.

Worth, Guy C. (2) 174.

Worthington, George (3) 119.

Wright, Benoni (3) 25.

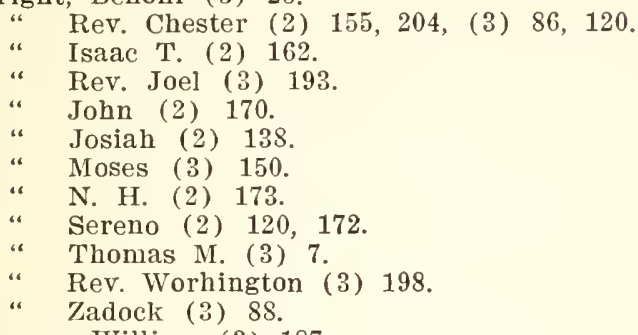

Wyman, William (3) 187.

Y.

Yale, Rev, Calvin (2) 149, (3) 51.

Yates, Robert (2) 83.

York, Duke of (2) 18, (3) 3.

Young, - (2) 216.

“ Ammi B. (2) 130, 131, 132, (3) 120.

“ Augustus (2) 223.

“ Rev. Dan (2) 181.

“ Sir George (3) 3.

“ Rev. Joab (3) 166.

“ John (3) 199.

“ Thomas (1) 4, (2) 51, 52, 105, 106.

“ Rev. Zebina (2) 188. 

General Subject Index 



\section{General Subject Index}

A.

"A" punishment by marking with (2) 121.

Academies (2) 143-4.

Black River Academy (2) 188.

Burr Seminary (2) 168.

Derby Institution (2) 188.

Leland English and Classical Institute (2) 188.

Moor's Charity School, N. H. (2) 144.

Newbury Seminary (2) 184.

Orange County (3) 146.

Troy Conference Academy (2) 184.

Vermont Literary and Scientific Institution (2) 188.

Agriculture (2) 213.

Allegiance, Oath of (3) 171.

Alien and Sedition Laws (2) 89.

Area of Vermont (1) 2.

Arsenals (2) 128.

Aurora Borealis (1) 18-19.

B.

Banks (2) 91, 92, 101-3, 122-133, 137.

Burlington, (2) 135.

Essex (2) 137.

Middlebury (2) 136-7.

Windsor (2) 135 .

Woodstock (2) 136.

Battles (3) 59, 173.

Bennington (2) 44, 45, 46, 128.

Bunker Hill (2) 7, (3) 7 .

Fort Edward (2) 9.

Fort William Henry (2) 10-11.

Hubbardton (2) 128 .

Lake Champlain (2) $34,39$.

Lake George (2) 9.

Otter Creek (2) 39.

Plattsburg (2) 33.

Stillwater (2) 47.

Ticonderoga (2) 12-13, 33-4, 40-2, 66.

Killed in Battles (2) 96-7.

"Beech Seal" (2) 31-2, 120. 
Birds of Vermont (1) 56.

Block Houses and Forts (2) 69.

On Otter Creek (2) 25.

On Winooskl (2) 26-7.

Bridgman's Fort (2) 68.

Boats

Blacksnake (2) 95, 121.

Carleton (2) 38.

Commodore Preble (2) 95.

Eagle (2) 95.

Frances (2) 95.

Growler (2) 95.

Inflexible (2) 38.

Maria (2) 38.

Montgomery (2) 95.

President (2) 95.

Royal George (2) 64 .

Thunderer (2) 38.

Books Printed in Vermont (2) 173.

Botany (1) $173-224$.

Bridges (3) 58, 151.

Natural (3) 197.

Boundary of Vermont (1) $1-2$.

Disputes

Vermont and Massachusetts (2) 17, 56.

Vermont and New Hampshire (2) 17, 52-6, 61, 73, 83 .

Vermont and New York (2) 19-32, 44-9, 51-3, 61, 72, 73, $75,76,83$. Also see under Congress.

British, Negotiations with (2) 61-7.

C.

Canada (2) 101.

Agent sent to (2) 66 .

Assists Americans (2) 35-6.

Invasion of (2) 35-9.

Rebellion in (2) 103.

Troops of (2) 48.

Trouble with (2) 90, 94-5.

Canals (2) 67, 99-100, 215-17.

Castleton Medical College (2) 160-5.

Catamount Sign, Bennington (2) 32, (3) 18 .

Caves (3) 39, 65, 85, 109.

Clarendon (1) 8.

Lake Dunmore (3) 158.

Plymouth (1) 8, (3) 141-2.

Census (2) 30.

Charters of Towns, Form of (2) 224. 
Children Lost (3) 169.

Churches (2) 175-204.

Baptists (2) 184-8.

Freewill (2) 188-90.

Catholic (2) 201-2.

Christian (2) 190-3.

Congregational (2) 176.

Dorrilites (2) 202.

Episcopal (2) 194.

Friends (2) 193.

Methodists (2) 180-4.

New Lights (2) 204.

Pilgrims (2) 203.

Unitarian (2) 189.

Universalists (2) 192-4.

Climate (1) 9.

Cold Weather (2) 102.

Colleges

Dartmouth College (2) 144-5, 197.

Middlebury College (2) 91, 152-61.

Norwich University (2) 168-71.

Vermont Academy of Medicine (2) 160-5.

Vermont Medical College (2) 165-7.

University of Vermont (2) 144-52.

Colonial Wars (2) 1-16.

Colored people in Vermont (2) 211.

Common Schools

see Schools, public.

Congress (2) $34,43$.

and Boundary dispute (2) 49, 51, 52, 57-60, $71-7$.

Connecticut,

Emigrants from (2) 121.

Loan from (2) 33.

Constitution

United States (2) 89.

Vermont (2) 52, 53, 102, 105-16.

Continental Congress, see Congress.

Money (2) 133.

Conventions,

Albany, N. Y. (2) 7-8, 21.

Brattleboro (2) 5-6.

Cornish, N. H. (2) 55.

Dorset (2) 49-50.

Hartford, Ct. (2) 97.

Manchester (2) 25.

Philadelphia, Pa. (2) 28.

Presidential (2) 104.

Wells (2) 79 . 


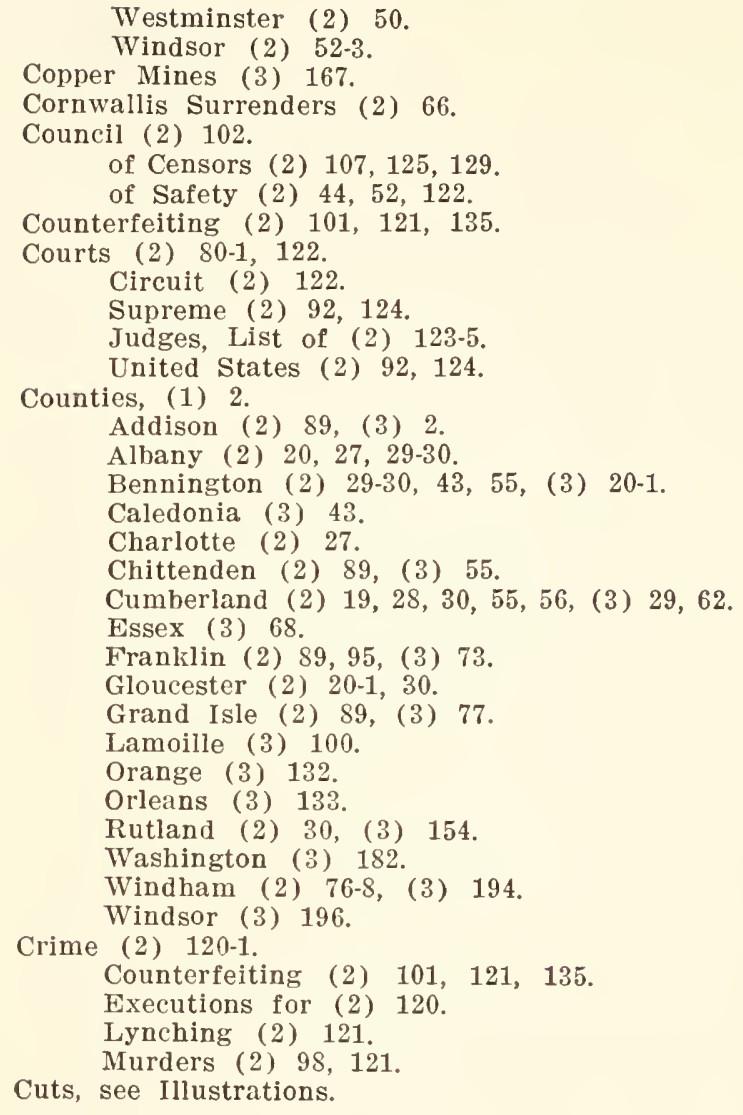

D.

Dark Days (2) 15-16.

Dartmouth College (2) 144-5, 189, 197.

Debt, in Prison for (2) 101.

U. S. Public (2) 78.

Deerfield Massacre (3) 192.

Deposit, Money (2) 143. 
Diseases, Vt. (2) 220-22.

Dutch Settlers, Vt. (3) 13.

E.

Early Settlers (2) 212.

Earthquakes, Vt. (1) 16-17.

Education, see Academies, Colleges, and Schools. Vermont (2) 141-74.

Elections (2) 53, 103-4.

Epidemics (2) 220. 1811-12 (3) 7, 9, 10, 23, 51, 52, 57, 68, 73, 87, 199.

Expeditions Across Vt. (2) 5 . Rogers (2) 14-15.

F.

Falls, (3) 77, 88-9, 128, 164.

New Haven (3) 128, 174, 176, 196.

North Hero (3) 129.

Orwell (3) 133.

Families, Large (3) 93, 191-3.

Sabin, Mrs. Gideon (3) 86 .

Financial Conditions (2) 79 .

Legislation (2) 81.

Forts (2) 129-30, (3) 13, 17, 176, 179.

Barnard (2) 68, (3) 24.

Bridgeman (2) 207.

Castleton (2) 68.

Cedars (2) 37.

Chambly (2) 35-6.

Corinth (2) 68 .

Crown Point (2) 34-5, 38-9.

Dummer (2) 16-17, 68.

Edward (2) 42-3.

Hoosic (2) 68, (3) 13.

Newbury (2) 68 .

Peacham (2) 68.

Pittsford (2) 68, (3) 139.

Putney (3) 145.

Rutland (3) 153.

Sorel (2) 38.

Stanwix (2) 47.

Ticonderoga (2) 33, 38-40.

William Henry (2) 10.

France, War with (2) 7-16, 88-9.

Free Speech (2) 102.

Freshets (2) 93. 
G.

Geography, Physical (1) 1.

Geological Survey (2) 104

Geology (1) 222-4.

Granite (3) 11.

German Settlers (3) 89, 160.

German Troops (2) 40, 45.

Gerrymandry (2) 90.

Government, State (2) 75-6.

Great Britain, Negotiations with (2) 59-61, 72, 74 .

Governors, Election of (2) 97.

Roster of (2) 118.

Votes for (2) 119.

and Council (2) 58, 76-8.

Lieutenant, Roster of (2) 118.

Granite (3) 11.

Green Mountains, Deseription of (1) 3 . Diagram of (1) 3 .

Green Mountain Boys (2) 23, 33, 96-7, 128, (3) 17, 158. Tavern, Bennington (2) 32 .

H.

Hazens Road (2) 68-9, 71, (3) 104.

Health, Public (2) 220.

High Bridges (3) 39.

High Schools, see Schools.

Highways (2) 18, 68-9, 222, (3) 136, 153.

Holy Rollers, see Churches, New Lights.

House of Representatives, see Legislature, House.

Hubbardton, Battle of (3) 92 .

Hunters (3) 111.

I.

Illustrations

Battle, Bennington (2) 46 .

Hubbardton (2) 43.

Bills and Coins (2) 134-5.

Burlington Harbor (2) 217.

Plan of Village (3) 39.

Burr Seminary (2) 168.

Church, Burlington (2) 201, (3) 38 .

Brandon (2) 185

Woodstock (2) 190, 192.

Clarendon, Map of (3) 56 .

Flag of Vermont (2) 128. 
Fort Bridgeman (2) 68 .

Indian (2) 205.

Relics (2) 207-8.

Montpelier, Map of (3) 121.

Punishment, Bennington (2) 31 .

State House (2) 130.

Prison (2) 138.

Troy Academy (2) 189.

University of Vermont (2) 144.

Vergennes (3) 175.

Woodstock Court House (2) 122.

\section{Indian}

Burial Places (2) 206-7.

Claims, Vt. (2) 89-91, 207.

Depredations (2) 67-71, (3) 6, 8, 24, 27, 29, 47, 79, 98, 101, $145-6,161,174,177,179-80,192-3$.

Barnard (2) 69 , (3) 17-18.

Bethel (3) 24.

Brandon (2) 69.

Capture of Caleb Howe and others (2) 68 .

Charleston, N. H. (2) 15, (3) 8.

Fort Bridgeman (2) 68.

Joe Johnson, Capture of (2) 71 .

Newfane (2) 68.

Newbury (2) 71 .

Pawlet (3) 136.

Randolph (2) 69.

Royalton (2) 69.

Sharon (2) 69.

Stockbridge (3) 112.

Windham Co. (2) 71.

First Settlement, Vt. (2) 205-8, (3) 113.

Hieroglyphics (2) 208.

Pequot War (2) 3.

Raids (3) 6-8, 27, 29, 47, 101, 145, 152, 177.

Relics (2) 207, (3) 179.

Summer (1) 16.

Indian Settlements (3) 113.

Indians (2) $1-16,35,40-1,45,47-8$.

St. Francis (2) 14-17, 205-6.

Industries (2) 213-14.

Encouraged (2) 98.

Iron Works (3) 11, 20.

Insane Asylums (2) 219.

Insurance (2) 219-20.

Iron Ores (3) 11, 20, 54, 57, 107, 117-18, 170, 176. 


\section{J.}

Judges (2) 122.

Justices of the Peace (2) 123.

$\mathrm{K}$.

Kentucky (2) 89.

L.

Lake and Ponds (1) 5-6.

Champlain (1) 2, 5-6, (2) 70, 95, (3) 48.

Bays in (1) 6 .

Basin Harbor (3) 12.

Bellemaquidder (3) 13.

McQuam (3) 112.

Missisco (3) 117.

South (3) 165.

Capes and Points in

Allen's (3) 3.

Pottier's (3) 142.

Sharpshin (3) 160.

Shelburne (3) 142.

Windmill (3) 3 .

Islands in (1) 5 .

Four Brothers (3) 72 .

Hog (3) 91.

Isle aux Noix (2) 34,63 .

Juniper (2) 216, (3) 100.

Rock Dunder (3) 65.

Sloop (3) 163.

Valcour (2) 39.

Steam Navigation in (2) 98.

Ponds, Dummer (3) 65.

Elligo (3) 67.

George (2) 215.

Hosmer (3) 60.

Joe's (3) 40.

Long (3) 74.

Memphremagog (2) 6, 99, (3) 112.

Molly's (3) 41.

Runaway (3) 74, 100.

Seymour (3) 121.

Willoughby (3) 188.

Laws (2) 103, 120.

Legislature, Vt. ( 2) 55, 57-8, 64, 73-5, 83, 88-94, 97-101, 117, 119.

House (2) 118. 
senate (2) 1.

Places of Meeting (2) 72-4, 77-9, 81, 119.

Preachers before (2) 102.

Legislation, see subject.

Liberty of Vermont, Vindicated (2) 58-9.

Libraries, Public (3) 56, 84.

Light Houses (2) 216-17.

Longevity (2) 222, (3) $3,24,52,68,77,80,88,90,93,139,149,165$, $170,173-5,179,189,191,193$.

Lotteries (2) 222.

Lynching (2) 121.

M.

Magnetic Variations (1) 19.

Manufacturing, see Industries.

Marble (3) 170.

Massachusetts (2) 44, 58, 78-9, 97, 133.

Claims, Vt. (2) 56 .

Massacre, Westminster (3) 188, 198.

Medical Colleges, see Colleges.

Societies (2) 167-8.

Medicinal Plants (1) 221.

Meteorology (1) 9-14.

Meteors (1) 16.

Middlebury College (2) 152-61.

Military Associations (2) 22.

Attacks, British (3) 137.

Expeditions (3) 136.

Militia (2) 21, 45, 47, 56, 63, 73, 76, 77-81, 96, 127-30, (3) 17, 104.

Mineral Springs (3) 191.

Mines (3) 141.

Missouri (2) 98 .

Money, Continental (2) 133.

Currency (2) 133-7.

Montreal (2) 35-8.

Moors Charity School (2) 144.

Mountain Peaks, Diagram of (1) 3.

Ascutney (3) 5 .

Camel's Hump (3) 44.

Defiance (2) 47.

Hope (2) 40, 47.

Independence (2) 129, (3) 123.

Killington (3) $100,162$.

Mansfield (3) 110.

Nebo (3) 123.

Shrewsbury (3) $100,162$.

Tom (3) 124 
N.

Natural Bridge, Wolcott (3) 197.

New Hampshire, Controversy with (2) 17-18.

North Carolina (2) 92.

Norwich University (2) 102, 168-71.

O.

Old Tenor Currency (2) 133.

Orange County Grammar School (3) 147.

Ordnance in Vermont, 1841 (2) 129.

Ordnance Corps, U. S. (2) 129.

Otter Creek, Affair at (2) 24-5.

P.

Paint Deposits (3) 89 .

Patriots' War (2) 103.

People Large Stature (3) 139.

Periodical (2) 174.

Episcopal Register (2) 200.

Pennsylvania Packet (2) 65 .

State Banner (2) 24, 32.

Vermont Register (2) 175.

Voice of Freedom (2) 218.

Pensions (2) 100.

Physicians (3) 110.

Political Parties (2) 85, 102.

Anti-Masonic (2) 100-1.

Anti-Slavery (2) 104.

Federalist (2) 88, 98-9.

Polygamy (2) 120-1.

Ponds (1) 5-6. See also Lakes and Ponds.

Population (2) 29-30, 208-12, (3) 15.

Post Office (2) 223.

Preachers before the Legislature (2) 102 .

Presidential Conventions (2) 104.

Vote (2) 104.

Printing (2) 171-2.

Prison, State (2) 138-9.

Prisoners, Captured (2) 34, 36, 37, 46-7, 96.

Q.

Quebec, Attack on (2) 36, 37, (3) 137. 
R.

Railroads (2) 88, 101, 217.

Regulators in Rutland (2) 81.

Representatives in Congress (2) 223.

House of, Vt., see Legislature, House.

Reptiles of Vt. (1) 112-27.

Revenue, State (2) 139-40.

Revolutionary War (2) 33-71.

Riots,

Hartland (2) 80.

Rutland (2) 80-1.

Westminster (2) 2S-9.

Windsor County (2) 76-8.

Windsor (2) 80-1.

Rivers and Streams (1) 4-5.

Barton (3) 12.

Battenkill (3) 12.

Black Creek (3) 25.

Black (3) 25.

Brown's (3) 36.

Castleton (3) 47.

Clyde (3) 55 .

Connecticut (2) 18, 69, 99, 216, (3) 58.

Deerfield (3) 63.

Dog (3) 64 .

Farrand's (3) 71.

Green (3) 78 .

Hoosic (3) 79.

Hubbardton (3) 93.

Huntington (3) 94.

Indian (3) 95.

Joe's (3) 99.

Lamoille (3) 74 .

Laplot (3) 101.

Leech's (3) 102.

Lemonfair (3) 103.

Lewis Creek (3) 103.

Little Otter Creek (3) 103.

Locust Creek (3) 103.

Mad (3) 108.

McQuam Creek (3) 112.

Merritt's (3) 99.

Middlebury (3) 115.

Miles (3) 116.

Mill Brook (3) 115.

Miller's (3) 116.

Missisco (3) 117.

Moose (3) 121. 
Muddy Brook (3) 124.

Nulhegan (3) 131.

Ompompanoosuc (3) 131.

Onion, see Winooski.

Otta Quechee (3) 134.

Otter Creek (2) 96, 129, (3) 134.

Passumpsic (3) 135.

Pawlet (3) 136.

Pike (3) 22.

Platt, see Laplot R.

Poultney (3) 144.

Quechee, see Otta Quechee.

Richelieu (2) 36.

Rock (3) 150.

Saxtons (3) 159.

Stevens Branch (3) 9-10.

Stevens (3) 165.

Trout (3) 173.

Walloomscolk (2) 20, (3) 180.

Wantasticook (3) 180 .

Waterbury (3) 183.

Wells (3) 186.

West (3) 180.

Whetstone Brook (3) 140 .

White Creek (2) 69, (3) 190.

White (2) 69, (3) 190.

Wild Branch (3) 191.

Williams (3) 191.

Willoughby's (3) 192.

Winhall (3) 197.

Winooski (2) 70, (3) 197.

Rogers Expedition (2) 14-15.

Royalton, Burning of (3) 7 .

Rum, Explosion of (3) 192.

$\mathrm{S}$.

St. Armand, Attack on (2) 95.

St. Johns, Abandoned by American Army (2) 38.

Siege of (2) 35-6.

Surrender of (2) 36 .

Salary, State Officers (2) 140-1.

School Fund (2) 142.

Statistics (2) 211.

Schools, Private, see Academies.

Public (2) 100, 141-75.

High (2) 143-4.

Scotland, Settlers from (2) 24-6, 212, (3) 154-5, 176.

Seal, Vermont (2) 107, 133. 
Seasons (1) 13.

Seasons, Remarkable (1) 20.

Secretaries, Vermont (2) 118.

Senate, Vt. (2) 102, 125.

Senators, Roster of (2) 223.

Settlers, Early (2) 29-30, 212.

Settlement, Bennington (3) 15 . Vermont (2) 20-32.

Shay's Rebellion (2) 78-9.

Shire Towns (1) 2.

Silk, Growing of (2) 102.

Slavery (2) 218.

Slave Trade (2) 102.

Slaves, Importation of (2) 92.

Smallpox, Epidemic of (1) 38 .

American Army (2) 37.

Smuggling (2) 95, 121.

Snow Fall, 1816 (2) 98.

Societies, American Colonization (2) 98.

Soil, Description of (1) 6-7.

South Carolina (2) 100.

Spafford's Gazetteer of New York (1) 14.

Speakers, House of Representatives (2) 118.

Springs, Medicinal (1) 7, (3) 192.

State, Vermont, Governors (2) 118.

House (2) 101, 130-2, (3) 175.

Insane Asylum (2) 219.

Legislature not to decide on Constitutionality of the law (2) 89.

Lieutenant-Governors (2) 118.

Prison (2) 93, 138-9.

Seal (2) 107, 133.

School Statistics (2) 211.

Treasurers (2) 118.

Steamboat, Companies and Captains (2) 215.

Steamboats (2) 215-17.

Steam Navigation (2) 98, 215-17.

Sugar, Maple (3) 58.

Sunday Schools (2) 187.

Surveys (3) 13, 91.

T.

Taxation (2) 105.

Taxes (2) 103.

Torles (2) 48.

Town Surveys (2) 18, 21.

Townships (2) 224. 
Towns, Vermont.

Acton (3) 1.

Addison (3) 1-2.

Aikens Gore (3) 2.

Alburg (3) 3.

Andover (3) 3 .

Arlington (2) 31, (3) 4-5.

Athens (2) 70, (3) 5 .

Averill (3) 6.

Avery's Gore (3) 6.

Bakersfield (3) 7 .

Baltimore (3) 7 .

Barnard (2) 28, (3) 7, 24.

Barnet (3) 8.

Barre (3) 9-10.

Barton (3) 12.

Bellows Falls, see Rockingham.

Belvidere (3) 13.

Bennington (2) $17,23,32,83,121,128,176$, (3) 13-22. Conventions at (2) 21.

Irish Corner (3) 20.

Benson (3) 21-2.

Benton's Gore (3) 22.

Berkshire (3) 22.

Berlin (3) 22-3.

Bethel (3) 24.

Billymead, see Sutton.

Bloomfield (3) 25.

Bolton (3) 25 .

Bradford (3) 25.

Bradleyville (3) 26.

Braintree (3) 27.

Brandon (2) 69, (3) 27.

Brattleboro (2) 71, 77-8, (3) 28.

Conflicts at (2) 78 .

Bridgewater (3) 31.

Bridport (2) 33, (3) 31.

Brighton (3) 33.

Bristol (3) 34.

Bromley, see Peru.

Brookfield (2) 70 , (3) 34 .

Brookline (2) $70-1$, (3) 35 .

Brownington (3) 36 .

Brunswick (3) 36 .

Buel's Gore (3) 36 .

Burke (3) 36.

Burlington (2) 86, 91, 95, 99, 120, 121, 126, 129, (3) 37. Breakwater' (2) 217.

Cabot (3) 40.

Calais (3) 41 . 
Caldersburg, see Morgan.

Cambridge (3) 43.

Canaan (2) 95, (3) 44.

Carthage, see Jay.

Castleton (2) 41, 65, 68, (3) 45 .

Cavendish (3) 47.

Charleston (3) 50.

Charlotte (2) 20, (3) 51.

Chelsea (2) 121, (3) 52.

Chester (3) 52.

Chimney Point (3) 1.

Chittenden (3) 54.

Clarendon (2) 26, (3) 55.

Colchester (2) 86, (3) 56.

Winooski (2) 28, (3) 57.

Concord (3) 57.

Corinth (2) 71, (3) 58.

Cornwall (3) 58.

Coventry, name changed to Orleans.

" (3) 60 .

" Gore (3) 60 .

Craftsbury (3) 60 .

Cumberland Head (2) 95, (3) 97.

Danby (3) 62.

Danville (2) 92 , (3) 62.

Derby (3) 63 .

Deweysburgh, now parts of Danville and Peacham (3) 64 .

Dorset (2) 49-50, (3) 64 .

Convention at (2) 49-50.

Dover (3) 65 .

Dummerston (3) 65 .

Duncanborough, now Newport (3) 64 .

Durham (3) 66.

Duxbury (3) 66 .

East Haven (3) 66.

Eden (3) 66.

Elmore (3) 67.

Enosburg (3) 67.

Essex (3) 68 .

Fairfax (3) 69 .

Fairfield (3) 69.

Fairhaven (3) 70.

Fairlee (3) 70.

Fayettsville, see Newfane.

Fayston (3) 71 .

Ferdinand (3) 71 .

Ferrisburgh (3) 71

Flamstead, now Chester.

Fletcher (3) 72.

Franklin (3) 72 . 


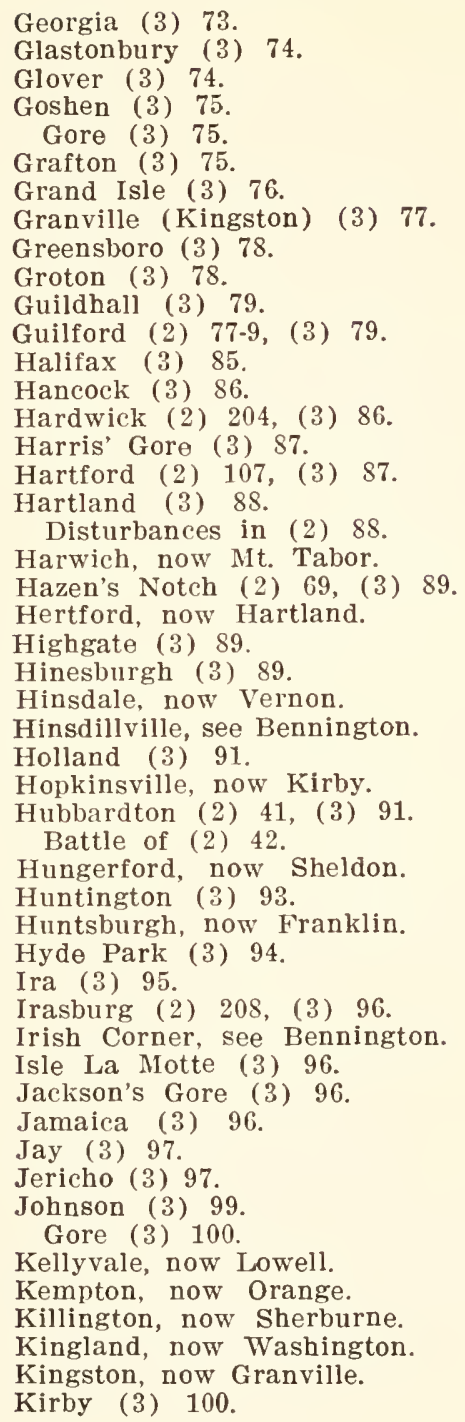


Knight's Gore, see Bakersfield.

Knowlton's Gore, see Bakerstield.

Landgrove (3) 101.

Leicester (3) 102.

Lemington (3) 102.

Lewis (3) 103.

Lincoln (3) 103.

Littleton, now Waterford.

Londonderry (3) 103.

Lowell (3) 104.

Ludlow (3) 104.

Lunenburg (3) 107.

Lutterloh, now Albany.

Lyndon (3) 108.

Lyndonville, see Lyndon.

Maidstone (3) 108.

Manchester (2) 44-5, 47, (3) 109.

Councll of Safety, meet at (2) 52.

Mansfield (3) 109.

Marlborough (3) 110.

Marshfield (3) 112.

Marvin's Gore (3) 112.

Medway (3) 112.

Middlebury (2) 90, 92, 125, (3) 113.

Middle Hero, now Grand Isle.

Middlesex (3) 115.

Middletown (3) 115.

Milton (3) 116.

Minden, now Craftsbury.

Minehead, now Bloomfield.

Missisco, now Troy.

Monkton (3) 116.

Montgomery (3) 118.

Monroe (3) 119 , now Woodbury.

Montpelier (2) 99, 120, 125-6, (3) 119.

Becomes the Capital (2) 92, 130.

Moretown (3) 121.

Moretown, old name for Bradford (3) 25 .

Morgan (3) 121.

Morristown (3) 122.

Mt. Holly (3) 123.

Mt. Tabor (3) 123.

Neshobe, now Brandon.

Newark (3) 124.

Newbury

Newfane (2) 68, 71, (3) 126.

New Flamstead, now Chester.

New Haven (2) 198, (3) 127.

New Huntington, now Huntington.

Newport (3) 128. 


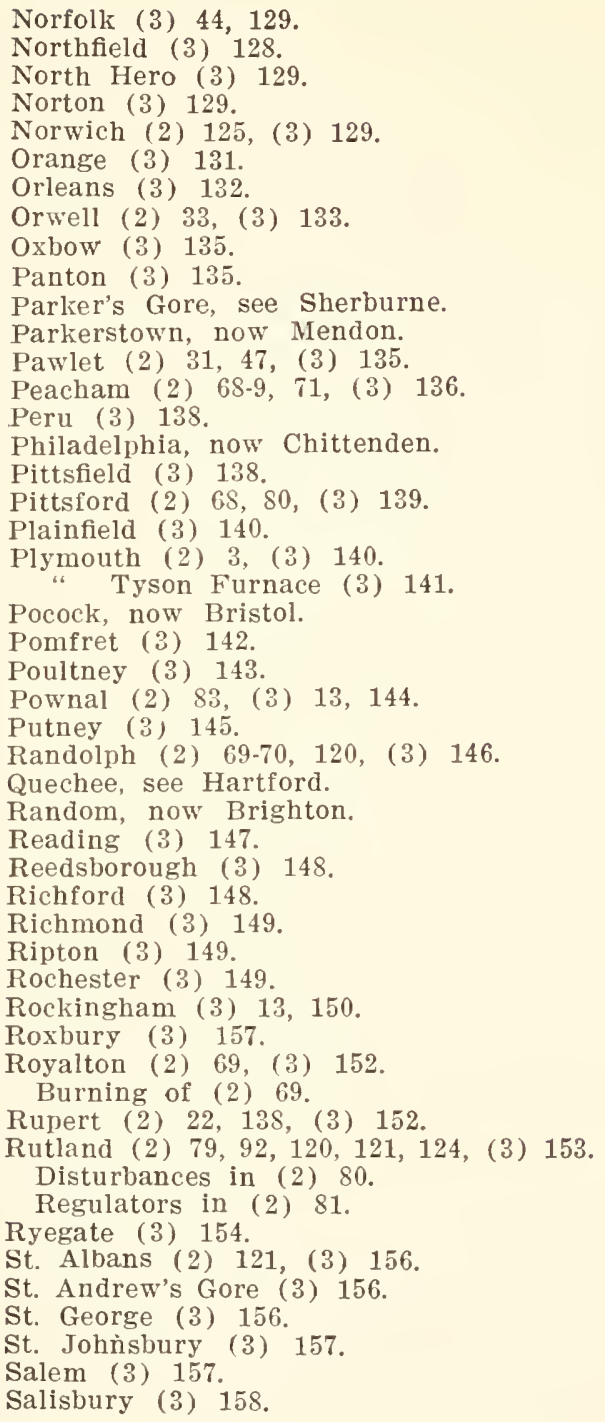


Saltash, now Plymouth.

Sandgate (3) 159.

Searsburg (3) 159.

Shaftsbury (2) 121, (3) 159.

Sharon (2) 69, (3) 160.

Sheffield (3) 160.

Shelburne (3) 160.

Sheldon (3) 161.

Sherburne (3) 161.

Shoreham (3) 162.

Shrewsbury (3) 162.

Smithfield, now Fairfax.

Socialborough, now Clarendon.

Somerset (3) 163.

South Hero (3) 163.

Springfield (3) 163.

Stamford (3) 164.

Starksborough (3) 164.

Sterling (3) 165 .

Stockbridge (3) 165 .

Stowe (3) 166.

Strafford (2) 188, (3) 166.

Stratton (3) 168.

Stratton's Gore (3) 168.

Sudbury (3) 168 .

Sunderland (2) 32, 64, (3) 168

Sutton (3) 169.

Swanton (3) 170.

Thetford (3) 170 .

Tinmouth (3) 171 .

Tomlinson, now Grafton.

Topsham (3) 172.

Waits River (3) 179.

Townshend (3) 172.

Troy (3) 173.

Tunbridge (2) 69, (3) 174.

Turnersburg, now Chelsea.

Twingsville, now Barre.

Tyson Furnace, see Plymouth.

Underhill (3) 175.

Vergennes (2) 88, 128, (3) 175.

Vernon (2) 17, (3) 176.

Vershire (3) 177.

Victory (3) 177.

Vineyard, now Isle La Motte.

Virgin Hall, see Aiken's Gore.

Waitsfield (3) 178.

Wait's River, see Topsham.

Walbridgeville, see Bennington.

Walden (3) 179. 
Wallingford (3) 179.

Wallumschoik (3) 15.

Waltham (3) 180.

Wardsborough (3) 180.

Warner's Gore (3) 181.

Warren (3) 181.

Warren's Gore (3) 181.

Washington (3) 181.

Waterbury (3) 182.

Waterford (3) 183.

Waterville (3) 183.

Weathersfield (3) 184.

Wells (2) 79, (3) 185.

Wenlock (3) 186.

West Fairlee (3) 186.

Westfield (2) 69, (3) 186.

Westford (3) 187.

West Haven (3) 187.

Westminster (2) 91, (3) 187.

Convention (2) 50 .

Massacre (2) 28-9.

Westmore (3) 188.

Weston (3) 188.

Weybridge (3) 189.

Wheelock (3) 189.

Whiting (3) 190.

Whitingham (3) 190.

Wildersburg, now Barre.

Williamstown (2) 107, (3) 192.

Williston (2) 86, (3) 192.

Wilmington (3) 193 .

Windham (3) 193.

TVindmill Point (2) 129.

Windsor (2) 80, 91, 92, 99, 105, 120, 124, 125, (3) 194. Convention (2) 52-3. Riots (2) $80-1$.

Winhall (3) 196.

Winooski (Colchester) (2) 28, (3) 57.

Wolcott (3) 197.

Woodbury, name changed to Monroe.

Woodford (3) 198.

Woodstock (2) 92-3, 121, 192, 203, (3) 198.

Worcester (3) 199.

Names Towns Changed (3) 200.

Towns other than Vermont.

Canada,

Chambley (2) 38.

Lacolle (2) 96.

St. John's (2) 34 .

Three Rivers (2) 37. 


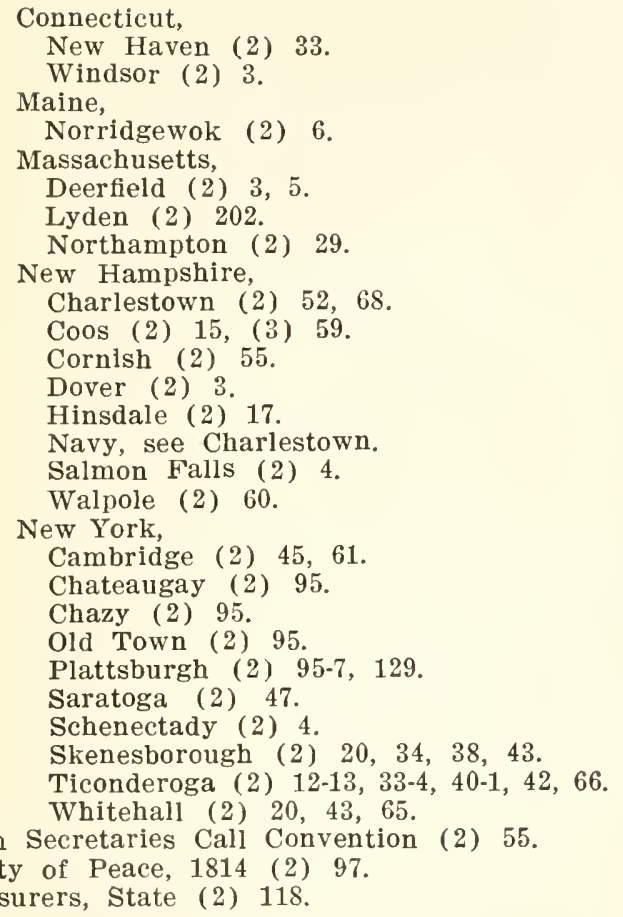

U.

Union with New Hampshire Towns (2) 60 .

New York Towns (2) 60.

University of Vermont (2) 144-52.

V.

Vermont

Admitted to the Union (2) $71,81$.

A Royal Province, proposition for (2) 2s-9.

Asks New Hampshire and Massachusetts for aid (2) 52-3.

Civil Policy of (2) 49.

Constitution (2) 105.

Declaration of Independence (2) 50-1.

Division of (2) 56 .

Emigrants from (3) 21. 
First Settlement of (2) 16-17, 43, 212, (3) 176.

Lays Claims to Towns in New Hampshire and New York (2) $60-1$

Origin of Name (1) 4 .

Order Restored in (2) 61.

Previous to 1760 (2) $16-17$.

Settlers (2) 28.

Territory, outline of (2) 54-5.

Royal Decision (2) 53-4.

Towns in Eastern Vt., withdraw (2) 55.

Vermont Academy of Medicine (2) 160-5.

Anti-Slavery Society (2) 218.

Bible Society (2) 187, 217-18.

Medical College (2) 165-7.

Mutual Insurance Co. (2) 219.

Virginia (2) 89.

W.

War of 1812 (2) 94-5, 130.

Whipping, public (2) 120 .

Women of Vermont (2) 70, 212.

Woodstock Medical College (2) 165-7.

Y.

Yankee Doodle (2) 48.

"Yorkers," Treatment of (2) 78-9.

Z.

Zoology of Vermont (1) 23-173. 







